University of Windsor

Scholarship at UWindsor

\title{
A Meta-analysis of Cognitive Functioning in Non-Demented Adults with Type 2 Diabetes Mellitus
}

\author{
Michelle Cécile Émélie Monette \\ University of Windsor
}

Follow this and additional works at: https://scholar.uwindsor.ca/etd

\section{Recommended Citation}

Monette, Michelle Cécile Émélie, "A Meta-analysis of Cognitive Functioning in Non-Demented Adults with Type 2 Diabetes Mellitus" (2012). Electronic Theses and Dissertations. 5540.

https://scholar.uwindsor.ca/etd/5540

This online database contains the full-text of PhD dissertations and Masters' theses of University of Windsor students from 1954 forward. These documents are made available for personal study and research purposes only, in accordance with the Canadian Copyright Act and the Creative Commons license-CC BY-NC-ND (Attribution, Non-Commercial, No Derivative Works). Under this license, works must always be attributed to the copyright holder (original author), cannot be used for any commercial purposes, and may not be altered. Any other use would require the permission of the copyright holder. Students may inquire about withdrawing their dissertation and/or thesis from this database. For additional inquiries, please contact the repository administrator via email (scholarship@uwindsor.ca) or by telephone at 519-253-3000ext. 3208. 
A Meta-analysis of Cognitive Functioning in Non-Demented Adults with Type 2 Diabetes

$$
\text { Mellitus }
$$

by

Michelle C. E. Monette

A Thesis

Submitted to the Faculty of Graduate Studies through Psychology

in Partial Fulfillment of the Requirements for the Master of Arts at the University of Windsor

Windsor, Ontario, Canada 2012

(C) 2012 Michelle C. E. Monette 
A Meta-analysis of Cognitive Functioning in Non-Demented Adults with Type 2 Diabetes Mellitus

by

Michelle C. E. Monette

APPROVED BY:

Dr. L. Patrick

Department of Nursing

Dr. D. Jackson, Co-Advisor

Department of Psychology

Dr. A. Baird, Co-Advisor

Department of Psychology

Dr. J. Casey, Chair of Defense

Department of Psychology

27 August 2012 


\section{DECLARATION OF ORIGINALITY}

I hereby certify that I am the sole author of this thesis and that no part of this thesis has been published or submitted for publication.

I certify that, to the best of my knowledge, my thesis does not infringe upon anyone's copyright nor violate any proprietary rights and that any ideas, techniques, quotations, or any other material from the work of other people included in my thesis, published or otherwise, are fully acknowledged in accordance with the standard referencing practices. Furthermore, to the extent that I have included copyrighted material that surpasses the bounds of fair dealing within the meaning of the Canada Copyright Act, I certify that I have obtained a written permission from the copyright owner(s) to include such material(s) in my thesis and have included copies of such copyright clearances to my appendix.

I declare that this is a true copy of my thesis, including any final revisions, as approved by my thesis committee and the Graduate Studies office, and that this thesis has not been submitted for a higher degree to any other University or Institution. 


\begin{abstract}
There is a large body of literature confirming the presence of cognitive functioning deficits in individuals with Type 2 Diabetes Mellitus (T2DM) who have not been diagnosed with dementia, but a clear pattern has yet to emerge. The main goal of the present study was to determine the pattern and magnitude of cognitive functioning deficits in individuals with T2DM without dementia using meta-analysis. Differences in cognitive functioning were evaluated between those with T2DM and non-diabetic controls on specified cognitive abilities as well as on individual neuropsychological tests. Individuals with T2DM performed significantly lower than non-diabetic controls $(p<$ 0.05) on all cognitive abilities and on all neuropsychological tests evaluated. The majority of effect sizes were in the small range. The findings from this study will help to inform clinical work with individuals with T2DM and will be a first step towards improving patient care and treatment adherence.
\end{abstract}




\section{ACKNOWLEDGEMENTS}

I wish to sincerely thank the people who supported and encouraged me to see this thesis through to completion. First and foremost, Dr. Anne Baird for her support and expertise, and for allowing me to pursue a project that was important to me even though this meant some extra work on her part, Dr. Dennis Jackson for his knowledge and help with the methodological aspects of the project, Dr. Linda Patrick for her indispensible comments and support throughout the proposal and defense process, and Katie Chauvin for her contributions to study coding.

I would like to thank Dr. Guy Proulx for being the first person to teach me about neuropsychology and how neuropsychology can be tied to diabetes research as well as the late Dr. Evelyne Corcos for first teaching me most of everything else I know about psychology. I want to thank my mom for her unwavering belief in me and continued support through my academic career. Lastly, I want to thank my sister for always giving me a fresh perspective on everything I do. 
TABLE OF CONTENTS

DECLARATION OF ORIGINALITY ......................................................................ii

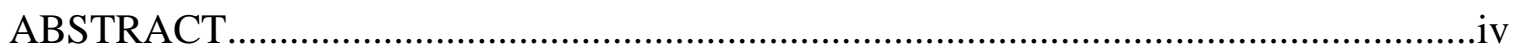

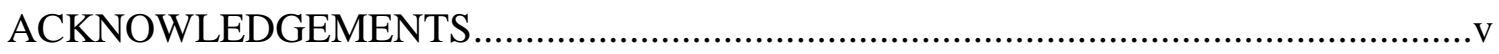

LIST OF

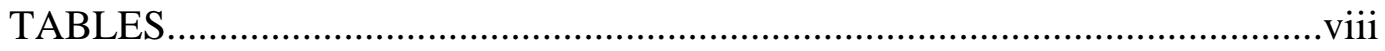

LIST OF

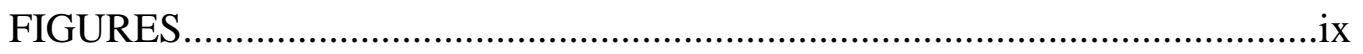

\section{CHAPTER}

I. INTRODUCTION AND REVIEW OF THE LITERATURE

Type 2 Diabetes Mellitus.......................................................................2

The Importance of T2DM, Cognitive Impairment, and Dementia...............4

The Cognitive Impairments Present in T2DM.............................................

Moderator Variables: Disease and Demographic Variables..........................

Chronic hyperglycemia..............................................................

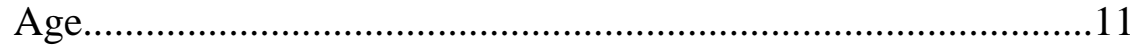

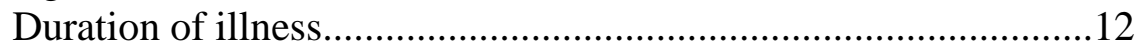

Treatment modality.....................................................................12

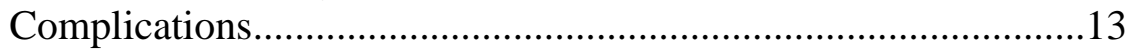

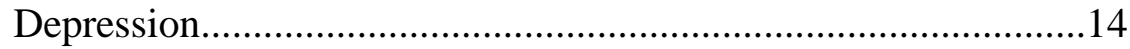

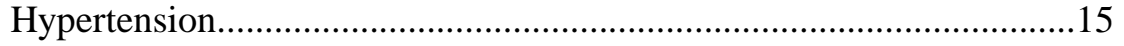

Moderator Variables: Methodological Variables........................................15

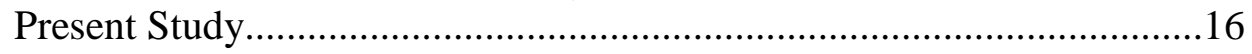

II. METHOD

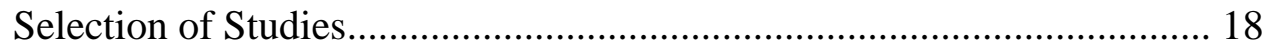

Search engines and search strategy............................................. 18

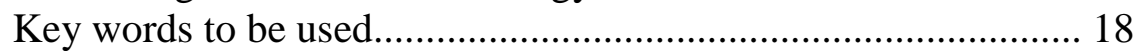

Inclusion and exclusion criteria............................................... 18

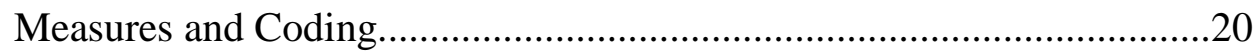

Cognitive ability classification...................................................20

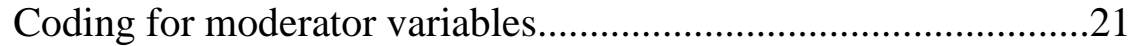

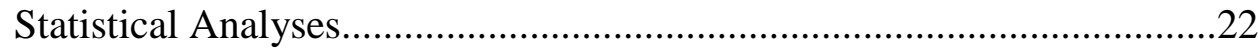

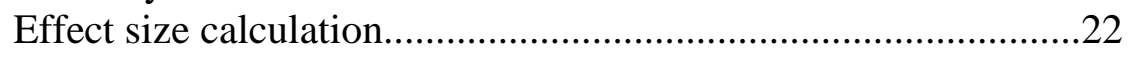

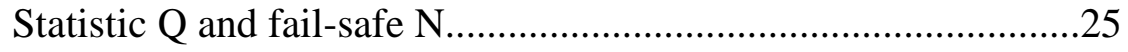




\section{RESULTS}

Article Inclusion in the Meta-Analysis................................................26

Heterogeneity and Publication Bias....................................................29

Diabetic Versus Non-Diabetic Controls: Comparison by Cognitive

Abilities.................................................................................... 31

Diabetic Versus Non-Diabetic Controls: Test-By-Test Meta-Analysis.....33

Moderator Variables Analyses.............................................................34

\section{DISCUSSION}

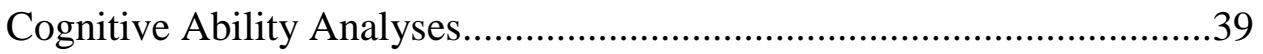

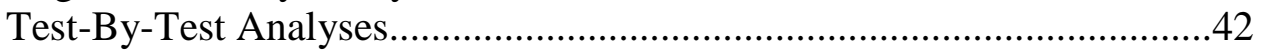

Moderator Variables Analyses..........................................................43

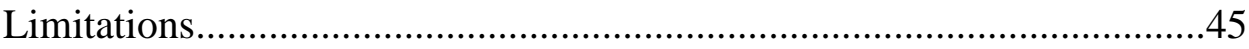

Future Research.............................................................................46

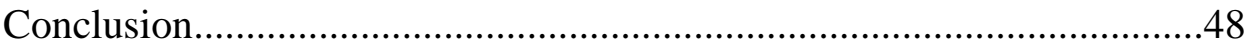

\section{APPENDICES}

Appendix A Glossary of Cognitive Abilities with Classification.........................50

Appendix B Meta-analysis Code Book...........................................................63

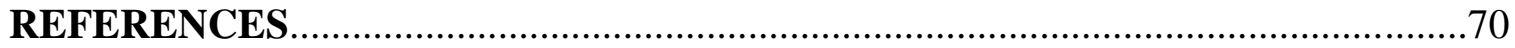

VITA

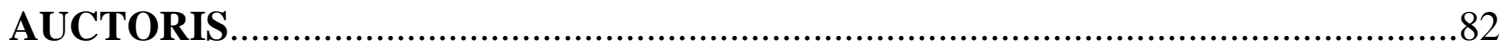




\section{LIST OF TABLES}

Table 1 Characteristics of the Twenty-Four Studies Included in the Meta-Analysis.......27

Table 2 Individual Cohen's $d$ s for Three Theoretical Moderator Variable Studies...........36

Table 3 Study Design and Publication Year Meta-Analyses.........................................38 


\section{LIST OF FIGURES}

Figure 1 Flow Chart of Article Inclusion..................................................................26

Figure 2 Average Cohen's $d$ and 95\% confidence intervals for cognitive abilities for diabetics versus non-diabetic controls comparison.................................................... 32

Figure 3 Average Cohen's $d$ and 95\% confidence intervals for selected neuropsychological measures for diabetics versus non-diabetic controls comparison.......34 


\section{CHAPTER I}

\section{INTRODUCTION AND REVIEW OF THE LITERATURE}

Many studies have reported modest deficits in multiple cognitive abilities in individuals with type 2 diabetes mellitus (T2DM) compared to nondiabetic controls (Awad, Gagnon \& Messier, 2004). However, there are differences between studies in the cognitive abilities found to be affected and the severity of cognitive deficits reported. The differences in study results are likely related to variability in demographic measures, T2DM characteristics, neuropsychological tests used to measure cognitive impairments, and study methodologies (Biessels, Deary \& Ryan, 2008; Brands et al., 2007).

Inconsistencies from one study to another in terms of the cognitive deficits associated with T2DM may arise from variations in what domains were assessed and how (Ryan \& Geckle, 2000b). There is also a selection bias towards healthy participants as many researchers are interested in preventing cognitive impairments in T2DM, with the consequence of excluding poorly controlled diabetics, diabetics with co-morbid conditions, and diabetics who are already cognitively impaired prior to the beginning of a study (Cukierman, Gerstein \& Williamson, 2005; Ryan, 2006). Consequently, the cognitive abilities affected in T2DM and the severity of cognitive deficits present are still unclear.

This review of the literature will encompass four sections. First, a brief overview of the etiology, complications, and treatments of T2DM will be given. Second, a review of the importance of T2DM to cognitive impairment and dementia will be elaborated. Third, a brief overview of the cognitive deficits most often present in T2DM, as well as findings from previous meta-analyses and reviews will be presented. Lastly, an overview 
of the findings linking the various disease and demographic variables to cognitive impairment in T2DM will be presented.

\section{Type 2 Diabetes Mellitus}

T2DM is the end result of a process that makes the body incapable of absorbing glucose because body tissues have become insulin resistant and can no longer produce enough insulin to keep glucose levels in the optimal range for proper metabolic functioning. Hyperglycemia (elevated blood glucose levels) is the indication of the presence of T2DM. Treatments for T2DM aim to keep blood glucose levels within the optimal range in order to prevent the complications of diabetes that result from chronically elevated blood glucose levels.

T2DM develops in stages that overlap (Defronzo, Bonadonna \& Ferrannini, 1997). First there is insulin resistance, a stage in which the tissues of the body become progressively more resistant to insulin. Age and obesity contribute to the development of insulin resistance and together account for $35 \%$ of the variance (Defronzo et al.). In the second stage, insulin resistance is followed by hyperinsulinemia (increased insulin production and secretion), which occurs to compensate for the insulin resistance and to maintain normal glucose levels. In the third and final stage, the body is no longer able to compensate as the cells of the body become so insulin resistant that the insulin producing beta-cells in the pancreas are no longer able to produce enough insulin to maintain glucose homeostasis.

When optimal fasting glucose levels can no longer be maintained (4.0 to 6.0 $\mathrm{mmol} / \mathrm{l}$ ), glucose intolerance (pre-diabetes) develops. This is a condition in which fasting blood glucose levels are elevated (equal or greater than $6.1 \mathrm{mmol} / \mathrm{l}$ ), but not as elevated as 
the levels found in overt T2DM (equal or greater than $7.9 \mathrm{mmol} / \mathrm{l}$ ). Finally, overt T2DM develops (Kumari, Brunner \& Fuhrer, 2000). The development of T2DM is related to increasing age, genetics, poor diet, physical inactivity, and obesity (Pradhan, 2007).

Treatment for T2DM is aimed at maintaining normal glucose levels in order to prevent the secondary complications of diabetes, including cardio- and cerebrovascular disease (atherosclerosis, heart attack, and stroke), nephropathy (kidney damage), retinopathy (damage of the small blood vessels of the retina which can lead to blindness), and neuropathy (peripheral nerve damage, that usually affects the legs and feet and can lead to infection and amputation; Brands et al., 2007). These complications result from chronically elevated blood glucose levels.

Initially, T2DM can be treated with lifestyle modifications including changes in diet, increased level of physical activity, and cessation of unhealthy behaviours such as smoking. In the beginning stages of the disease these changes can be sufficient to maintain target blood glucose levels. As the disease progresses, treatment with oral hypoglycemic drugs is first required, and as the disease progresses further the vast majority of type 2 diabetics need insulin injections to continue to achieve the target blood glucose levels needed to prevent complications related to the disease (Brands et al., 2007).

Whereas T2DM usually develops in middle to late adulthood, Type 1 Diabetes Mellitus (T1DM) typically develops in childhood or early adulthood. T1DM is caused by an autoimmune-mediated destruction of pancreatic $\beta$-cells. T1DM has a stronger genetic component than T2DM. The onset of T1DM is sudden, not gradual as in T2DM, and individuals with T1DM require multiple daily insulin injections to survive. Insulin 
injections are the only treatment option currently available to those with T1DM (Biessels et al., 2008).

\section{The Importance of T2DM, Cognitive Impairment, and Dementia}

Throughout the world, approximately 285 million people have been diagnosed with diabetes and seven million people receive a diagnosis of diabetes each year (CDA, 2011). This will raise the world prevalence to 438 million by 2030 (CDA). In Canada, more than 9 million people have diabetes or pre-diabetes (CDA). The proportion of individuals with T2DM is $90 \%$ as compared to $10 \%$ in T1DM (CDA).

The relative percentage of diabetics who are older than 65 is growing. In 2000, individuals 65 years of age and older made up $40 \%$ of all cases of diabetes in the United States; this percentage is expected to rise to $50 \%$ by 2025 , and $60 \%$ by 2050 (Boyle et al., 2001). Munshi et al. (2006) reported that cognitive dysfunction (defined by poor performance on neuropsychological measures) is present in $30-40 \%$ of diabetics 70 years of age and older.

Individuals with T2DM are almost two times more likely to develop dementia, and those with insulin-treated diabetes are four times more likely, when compared to nondiabetic controls (Ott et al., 1999). This difference in relative risk for dementia likely reflects the fact that the need for insulin injections is an indicator of more severe diabetes. It has been estimated that 7 to $13 \%$ of all cases of dementia can be attributed to diabetes (Biessels et al., 2008). Individuals with diabetes have a 1.2 to 2.3 times greater risk for Alzheimer's disease and a 2.2 to 3.4 times greater risk for vascular dementia than nondiabetics (Cukierman et al., 2005). 
These trends need not exist; the development of T2DM can be prevented (Hussain, Claussen, Ramachandran \& Williams, 2007), and therefore the cognitive deficits present in the disease are also preventable. T2DM is a modifiable risk factor for dementia: prevention and proper management of T2DM could lower the number of cases of dementia that develop in the population.

Because T2DM is a modifiable risk factor for dementia, it is imperative that we improve our understanding of the mechanisms that increase the risk of cognitive deficits in diabetes. This is necessary to enable the identification of the individuals with diabetes who are at the greatest risk of developing cognitive deficits to improve the management and prevention of cognitive deficits and dementia in these individuals.

\section{The Cognitive Impairments Present in T2DM}

The most common and severe cognitive impairments in T2DM are found in verbal delayed memory (Awad et al., 2004; Arvanitakis, Wilson, Bienias, Evans \& Bennett, 2004; Biessels et al., 2008) and processing speed (Awad et al.; Biessels et al.; Brands et al., 2007; Saczynski et al., 2008;). Awad and colleagues (2004) found that 35\% of studies reported delayed verbal memory impairments and $45 \%$ of studies reported processing speed impairments.

Findings on cognitive impairment in T2DM have been inconsistent for immediate memory (Awad et al., 2004; Biessels, van der Heide, Kamal, Bleys \& Gispen, 2002), nonverbal memory (Awad et al.; Cosway, Strachan, Dougall, Frier, \& Dreary, 2001), arithmetic (Awad et al.), verbal fluency (Awad et al.), and executive function (Awad et al.; Brands et al., 2007; Cosway, et al.). Visuospatial processing, long-term semantic 
memory, auditory and visual attention, and language abilities are usually found to be intact (Awad et al.).

A recent longitudinal study found significant differences between individuals with T2DM and controls on measures of information processing speed, attention, and executive functions at baseline and at follow-up four years later, but no differences on a composite score of memory measured using verbal and nonverbal memory tests (van den Berg et al., 2010). This study and many others (see Awad et al., 2004) have found inconsistent results in the cognitive domains and abilities that show deficits in T2DM. A meta-analysis of recent methodologically sound studies is needed to aid in determining which cognitive abilities are most often impaired in T2DM and to what magnitude.

A meta-analysis of adults with T1DM found significant differences in overall cognition, intelligence, processing speed, psychomotor efficiency, visual and sustained attention, cognitive flexibility, and visual perception between diabetics and non-diabetic controls (Brands, Biessels, De Hann, Kappelle, \& Kessels, 2005). Learning and memory, divided and selective attention, and language did not differ significantly between diabetics and controls. The authors looked at the effects of three disease variables (microvascular complications, poor metabolic control, and severe hypoglycemic episodes) on cognitive abilities. Only microvascular complications were found to contribute to cognitive dysfunction (Brands et al.). A meta-analysis of children with T1DM reported a similar pattern of differences in cognitive functions between diabetics and non-diabetic controls, in that children with T1DM showed impairments in overall cognition, intelligence, processing speed, psychomotor efficiency, attention and executive function, academic achievement, and visual motor integration. Learning and memory did 
not show any significant differences between children with T1DM and non-diabetic controls (Gaudieri, Chen, Greer, \& Holmes, 2008).

The cognitive functions affected in T1DM are not all the same as those affected in T2DM (Awad et al., 2004, Biessels et al., 2008) due to differing etiologies, pathogenesis, and demographic factors in individuals with T1DM and in individuals with T2DM. Therefore, the results from meta-analyses of cognitive dysfunction present in T1DM do not generalize to T2DM and a meta-analysis of studies of the cognitive dysfunction in T2DM is needed to discern the pattern and magnitude of this dysfunction in T2DM as has been done with the meta-analyses of T1DM.

Two reviews in the last decade have looked at cognitive impairments in T2DM. Awad and colleagues (2004) reviewed studies examining the cognitive impairments in T2DM in research published in 2002 and earlier. Their conclusions are very important for our understanding of the impairments found in T2DM. However, their study was a review, not a meta-analysis. Meta-analysis will quantify differences in cognitive functioning between type 2 diabetics and non-diabetic controls. This information is needed to understand better the magnitude of cognitive dysfunction in T2DM.

A second review was conducted that looked at studies published before March 2008 (van den Berg, Kloppenborg, Kessels, Kappelle \& Biessels, 2009). This review looked at the individual effects of T2DM, hypertension, dyslipidemia, and obesity on cognition. Median effect sizes were calculated where possible for a small portion of the studies included in the review. T2DM was found to affect processing speed (Cohen's $d=$ -.40 ), attention (Cohen's $d=-.50$ ), and memory (Cohen's $d=-.30$ ) as broad cognitive domains. 
Van den Berg and colleagues (2009) classified studies according to very broad cognitive domains based on those specified by Lezak, Howieson, \& Loring (2004). Because of the classification method used, many studies with valuable information on cognitive functioning in T2DM were excluded from the review and from the effect size calculations because the data could not be classified into one of the specified cognitive domains.

The classification of cognitive abilities in the present study as opposed to cognitive domains, described below, allowed for a larger number of studies to be included in the meta-analysis and for more detailed information about the cognitive functioning in T2DM to be measured. In addition, many more studies have been published since March 2008 and the present study incorporated these new findings.

\section{Moderator Variables: Disease and Demographic Variables}

The presence of diabetes alone may not be sufficient to cause cognitive dysfunction, as not all Type 2 diabetics have clinically significant levels of cognitive dysfunction (Munshi et al., 2006). Many demographic and disease variables are associated with increased risk for cognitive impairment in type 2 diabetics. The specific contributions of these variables to cognitive dysfunction in T2DM are also unresolved. Within meta-analysis, these disease and demographic variables are among a larger group of variables known as moderator variables (Hunter \& Schmidt, 2004). In meta-analysis, analysis of moderator variables is intended to explain the heterogeneity found in individual studies to clarify why individual studies that focus on the same subject with the same research question often find different results. Moderator variables can be methodological (for example, study design and sampling method used) or theory driven. 
The theory driven moderator variables hypothesized to contribute to the heterogeneity of results in studies of cognitive dysfunction in T2DM include chronic hyperglycemia (elevated HbA1c), age, duration of illness, treatment modality, presence of diabetes complications, diagnosis of depression, and/or hypertension. Each will be discussed in turn.

Chronic hyperglycemia. Hyperglycemia (high blood glucose levels) is a cardinal feature of diabetes that has been shown to cause cognitive impairments (Saczynski et al., 2008; Kumari et al., 2000). Acute hyperglycemia has been demonstrated to have some important effects on cognitive function, although they are transient and normal cognitive function returns within 90 minutes of re-establishing normal blood sugar levels (Sommerfield, Dreary \& Frier, 2004). Acute hyperglycemia is a common occurrence in those with T2DM; it can affect information processing speed, working memory, and attention. Individuals with T2DM may experience transient cognitive deficits that can impact their daily functioning (Sommerfield et al.). The present study excluded studies that have looked at the effects of acute hyperglycemia on cognitive functioning because the goal of the current study was to establish the level of persistent cognitive impairment in T2DM, not the transient impairment that is caused by acute hyperglycemia.

Chronic hyperglycemia, which is measured with Hemoglobin A1c (HbA1c), has a negative impact on cognitive functioning that is not reversible. HbA1c is a straightforward blood test that measures blood glucose levels over a three month period. It measures the glucose levels in the oxygenated hemoglobin of red blood cells, which are replenished in the body, every three months (Manschot et al., 2006). The severity of cognitive impairment is directly related to poor glycemic control in T2DM (Munshi, et 
al., 2006). Ryan and colleagues (2006) demonstrated a moderate improvement in day-today cognitive functioning in those with T2DM without significant cognitive impairment when better control of glucose levels was achieved. However, alleviating chronic hyperglycemia did not reverse already present cognitive impairment (Awad et al., 2004; Kumari et al., 2000; Ryan et al.).

Chronic hyperglycemia has been shown to have many neurodegenerative effects on the brain. First, hyperglycemia accelerates the development of advanced glycation end-products (AGEs) in the brain above the levels seen in normal aging by exacerbating oxidative stress (Biessels et al., 2002). AGEs are considered to be markers of protein aging and occur normally through metabolism and aging (Whitmer, 2007). However, excessive levels of AGEs are associated with neuropathology. AGEs are thought to be one of the pathways through which chronic hyperglycemia leads to diabetic complications (Singh, Barden, Mori \& Beilin, 2001). AGEs are found in beta-amyloid plaques, a hallmark of Alzheimer's disease (AD), and have been shown to increase plaque deposition in AD brains (Kumari et al., 2000). AGEs impair neuronal functioning by causing apoptosis, calcium influx, and inhibition of oxidative phosphorylation (Munch et al., 1998). AGEs are also found in the hippocampus and in neurofibrillary tangles (NFT), another hallmark of AD (Vitek et al., 1994).

Chronic hyperglycemia reduces hippocampal synaptic plasticity and causes decreases in regional cerebral blood flow, abnormalities in brain uptake and metabolism of glucose, abnormalities in aminergic neurotransmitter pathways, depressed transport of choline and glucose across the blood-brain barrier, and diabetic complications (Ryan \& 
Geckle, 2000b). The reduction in hippocampal synaptic plasticity is reversible with proper glycemic control (Whitmer, 2007).

Age. Most type 2 diabetics do not begin to show cognitive decline until later in life. Those who show cognitive decline early on tend to have comorbid conditions and poorly controlled diabetes (Awad et al., 2004; Biessels et al., 2008; Bruce et al., 2008; Ryan \&Geckle, 2000b). For this reason, only studies focusing on participants with an average age of 50 years or greater, or those studies focusing on individuals above 40 years of age when the average age of participants was under 50 years, were included in the present study. This was done to reach conclusions about cognitive dysfunction in T2DM that reflect the levels likely to be seen in the type 2 diabetic population.

There could be a synergistic relationship between the effects of T2DM and the effects of aging on cognition and the central nervous system (Ryan \&Geckle, 2000b). Pirttila, Jarvenpaa, Laippala, and Frey (1992) found that 36\% of those who were less than 65 years of age with T2DM and hypertension had brain atrophy, compared to only $12 \%$ of those of the same age who did not have diagnoses of either T2DM or hypertension. Showing the effects of age, $71 \%$ of those with T2DM and hypertension who were 65 and older had brain atrophy compared to only $42 \%$ of those who did not have diagnoses of either T2DM or hypertension. Similarly, greater proportions of older individuals with T2DM alone, when compared to younger individuals with T2DM alone, would be expected to show brain atrophy (Ryan \& Geckle, 2000b). The brain's capacity to function optimally declines with age and declines further in the presence of T2DM (Ryan \& Geckle, 2000b). 
The cognitive decline in T2DM has been characterized as accelerated aging. That is, the patterns of decline observed in T2DM are similar to those seen in the normal aging process, but they occur earlier than expected (Keefover, 1998; Tisserand \& Jolles, 2003). It has been estimated that the effect of T2DM on the brain is equivalent to 3 years of aging (Okereke et al., 2008). Arvanitakis, Wilson, \& Bennett (2006) found that deficits in semantic memory in diabetics were equivalent to the effects of 14 years of aging, and impairments in processing speed were equivalent to 6 years of aging.

Duration of illness. The length of time an individual has had T2DM impacts cognitive functioning. Generally, when there is a longer duration of the disease there is a higher risk of cognitive impairment (Ebady, Arami, \& Shafigh, 2008; Sastre \& Grimley, 2003; Saczynski et al., 2008; van Harten et al., 2007). Studies that look at newly diagnosed T2DM versus T2DM with a longer duration have found T2DM duration to be strongly associated with worse cognitive performance and greater decline in general cognition, verbal memory and category fluency (Okereke et al., 2008). Compared to those with normal blood sugar levels, those with T2DM had poorer processing speed, and those with T2DM for greater than 15 years had poorer processing speed and executive functioning (Saczynski et al.). Lastly, T2DM duration has been related to atrophy and white matter lesion severity, after adjusting for age (Manschot et al., 2006). In addition to this main effect of duration of illness on cognitive impairment in T2DM, illness duration interacts with age and diabetic control, that is, whether or not the individual has experienced chronic hyperglycemia.

Treatment modality. Much more research is needed to properly evaluate the effects of different treatment modalities (diet and exercise, hypoglycemic medications, 
and insulin injections) on cognition. When studies examine the differences between treated (with any of the three treatment modalities) and untreated diabetes, individuals with treated diabetes outperform individuals with untreated diabetes because treated diabetes allows for better control of blood glucose levels (Bruce et al., 2008). When studies examine the cognitive functioning of individuals with diabetes based on the treatment modality they use to control their diabetes, those taking insulin injections have the greatest risk for cognitive impairment (Saczynski et al., 2008). Ott and colleagues (1999) found that individuals with insulin treated diabetes had a 4.7 times greater risk of cognitive impairment than non-diabetics, whereas individuals with newly diagnosed diabetes had only a 1.3 times greater risk. At the time the study by Ott and colleagues was done, those with newly diagnosed diabetes would typically have been treated with diet and exercise or with hypoglycemic medications, but not with insulin injections. Therefore the comparison between insulin-treated and newly diagnosed diabetics in this study (Ott et al., 1999) very likely is also a comparison between insulin-treated and non-insulintreated diabetes.

Complications. The presence of diabetic complications greatly increases the probability that cognitive impairments will be present (Saczynski et al., 2008). For example, Ryan (2006) found that diabetics with retinopathy showed cognitive decline over the course of the study, whereas diabetics without retinopathy showed no change. Retinopathy has also been linked to cortical atrophy (Manshot et al., 2007). It has been argued that cognitive impairments should be classified as a complication of T2DM (Cukierman, et al., 2005). Those with T2DM are at higher risk for macrovascular and microvascular damage, which cause cognitive impairments in their own right (Manshot et 
al.). Diabetics are at greater risk for macro- and microvascular damage because of the pathological effects of hyperglycemia on the body. Macrovascular damage leads to cardio- and cerebrovascular disease, which can directly cause cognitive impairment in T2DM, and microvascular damage is indexed by neuropathy, nephropathy, and retinopathy in T2DM (Saczynski et al.). The present study had the goal of including studies where retinopathy and atherosclerosis were the diabetes complications evaluated.

Depression. Individuals with diabetes are two times more likely than nondiabetics to develop depression severe enough to warrant clinical intervention (Anderson, Freedland, Clouse \& Lustman, 2001; Kolt \& Seaquist, 2008). As many as one in three individuals with diabetes may have depression symptoms severe enough to affect quality of life, impair functioning, affect adherence to medical treatments, affect glycemic control, and increase the risk of complications (Anderson et al.). Having both T2DM and depression increases the number of depressive episodes, worsens glucose control, and increases diabetic complications (Blazer, Moody-Ayers, Craft-Morgan \& Bunchett, 2002). Watari and colleagues (2006) measured cognitive deficits in those with T2DM and diagnosed major depression, in those with T2DM alone, and in normal controls. They found that those with both depression and T2DM had poorer attention, executive function, and information processing speed than normal controls. Although there were no statistically significant differences between those with both depression and T2DM and those with diabetes alone, the scores of those with diabetes alone on the measures of cognitive functioning were better than those of individuals with both depression and T2DM, but worse than those of individuals without depression or T2DM. There were no 
differences in $\mathrm{HbA} 1 \mathrm{c}$ between the diabetics with or without depression, suggesting some independent effects of depression.

Hypertension. Hypertension has been associated with changes in cognitive functioning on measures of attention, learning and memory, executive functions, visuospatial skills, psychomotor abilities, and perceptual skills (Waldstein, 2003). Most studies of the cognitive effects of T2DM statistically control for the presence of hypertension by including hypertension as a covariate, which sometimes alters the results (van Harten et al., 2007) and other times does not (Manschot et al., 2006). An important finding is that diabetics with hypertension have greater brain atrophy than hypertensives or diabetics alone (Parttila et al, 1992). Diabetics with hypertension have a greater chance of cognitive decline than diabetics without hypertension (Bruce et al., 2008; Hassing et al., 2004). Systolic blood pressure levels have been shown to mediate the relationship between T2DM and impairments in episodic memory and processing speed (McFall, Geall, Fischer, Dolcos \& Dixon, 2010).

\section{Moderator Variables: Methodological Variables}

In addition to the theory driven disease and demographic moderator variables, in the present study, separate meta-analyses were completed for three methodological variables. First, sampling method was evaluated by contrasting population studies to case-control studies. Second, study design was evaluated by contrasting longitudinal studies to cross-sectional studies. Lastly, study publication year was evaluated by contrasting studies according to the year in which data were published, or, in the case of longitudinal studies, the years in which baseline data were collected. Studies were classified as dating to 2000 to 2005 or to 2006 to 2012. Previous reviews on cognitive 
functioning in T2DM have found these variables to have differing effects across studies (Awad et al., 2004; van den Berg, et al., 2009).

\section{Present Study}

The first goal of the current meta-analysis was to determine the level of overall deficits in cognitive abilities in individuals with T2DM compared to non-diabetic controls and to identify the abilities in which those with T2DM showed the greatest deficits relative to non-diabetic controls to clarify the findings in the literature. Only studies in which diabetics had an average age of greater than 50 years were included. It was hypothesized that individuals with T2DM have specific impairments in some cognitive abilities but not others when compared to non-diabetic controls and that the abilities likely to show the greatest deficits were delayed verbal memory and processing speed (Awad et al., 2004).

The second goal was to identify the neuropsychological tests with the largest differences in effect sizes between diabetics and non-diabetic controls in order to inform clinicians about which tests to use to measure cognitive deficits in T2DM. The addition of routine screenings of cognitive functioning to T2DM treatment regimens has been advocated by several authors (Cukierman, et al., 2005; Ebady et al., 2008; Murthy, Jawaid \& Schulz, 2008; Sastre \& Grimley, 2003). In order to make routine screenings feasible, the tests that best measure cognitive dysfunction in T2DM need to be identified. The identification of these tests will be an important first step in improving diabetes patient care in order to reduce the risk for the development of cognitive impairment and dementia in T2DM. 
A third goal was, insofar as the data permitted, to evaluate the possible effects of single moderator variables including age, diabetes duration, HbA1c levels, insulindependent or non insulin-dependent status, presence of complications (atherosclerosis or retinopathy), presence of depression, presence of hypertension, sampling method, study design, and publication year. Those with diabetes who are older (Ryan \& Geckle, 2000b), have had diabetes longer (Ebady et al., 2008), have higher HbA1c levels (Munshi et al., 2006), are insulin-dependent (Saczynski et al., 2008), have complications (Manshot et al., 2007), have diagnoses of clinically important depression (Watari et al., 2006), and have diagnoses of hypertension (Bruce et al., 2008) were hypothesized to have greater deficits in cognitive functioning than those who do not have these factors. Participants recruited to studies with a case-control sampling method, with cross-sectional designs, and with a publication year of 2000 to 2005 were expected to have greater deficits in cognitive functioning than participants recruited to studies with a population sampling method, with longitudinal designs, and with a publication year of 2006 to 2012 (Awad et al., 2004; van den Berg, et al., 2009).

In conclusion, the overall purpose of the present study was to clarify and quantify the nature and pattern of cognitive deficits present in T2DM by comparing cognitive functioning in non-demented individuals with T2DM to that of non-demented and nondiabetic controls. 


\section{CHAPTER II}

\section{METHOD}

\section{Selection of Studies}

Search engines and search strategy. The MedLine Ovid search engine was used to identify studies of cognitive functioning in individuals with T2DM versus non-diabetic control participants or studies comparing different type 2 diabetic patient groups. The PsycInfo search engine then was used to locate any studies not found using the MedLine Ovid engine. Medline and PsycInfo were search for articles published from January $1^{\text {st }}$ 2000 up to May $3^{\text {rd }}$ 2012. Reference lists from reviews found in the initial search and from published studies included in the meta-analysis were then searched to identify additional studies.

Key words to be used. The following key words were used to search for relevant articles: cognition, attention, learning, memory, executive functioning, information processing, spatial, intelligence, neuropsychological, and neurocognitive in order to find studies that have measured cognitive functioning in some manner. These key words were combined with diabetes, type 2 diabetes, type 2 diabetics, age, depression, duration, complications, retinopathy, atherosclerosis, treatment modality, hypertension, and hyperglycemia. These words identified relevant studies on T2DM for the diabetic and non-diabetic control comparison and for the analyses of the moderator variables.

Inclusion and exclusion criteria. Article titles, abstracts, and full-text where necessary, were examined to establish whether studies fulfilled the following inclusion criteria: (a) human participants were used, (b) the study was published or available in English after 2000 inclusive, or was a longitudinal study in which baseline data was collected after 1995 inclusive, (c) the study included only adults with a mean age of 50 
years or who were over the age of 40 years and diagnosed with T2DM, (d) the study had defined diabetes and control groups, (e) the study assessed cognitive abilities with standard neuropsychological tests, (f) the study assessed cognitive abilities when blood glucose levels were in the normal range, $(\mathrm{g})$ the study reported original research in which test scores were presented for the experimental and control groups (means and standard deviations) or statistics such as the exact $t$ or $F$ values were given in order for effect sizes to be calculated, (h) participants from individual studies did not overlap with any other study included in the meta-analysis, and (i) the study excluded individuals with diagnosed dementia (see Figure 1).

Likewise, article titles, abstracts, and full-text where necessary, were examined to exclude studies based on the following criteria: (a) non-human subjects were used, (b) the study was published before 2000 in languages other than English or was a longitudinal study in which baseline data were collected prior to 1995 , (c) the study included children or adults with a mean age under 50 years or participants under the age of 40 years or who had diagnoses of T1DM or gestational diabetes, (d) the study did not assess cognitive abilities using standard neuropsychological tests or assessed functioning with measures other than standard neuropsychological tests of cognitive ability (eg., measures of sensory functioning, socioemotional adjustment, quality of life, and activities of daily living performance), (e) studies that assessed cognitive abilities when hypoglycemia or hyperglycemia were induced, (f) studies that were reviews and not original studies or that were original studies but did not report the necessary statistics to allow for calculation of effect sizes, (h) participants from individual studies overlapped with another study 
included in the meta-analysis, and (i) the study did not exclude individuals with diagnosed dementia.

\section{Measures and Coding}

Cognitive ability classification. Cognitive abilities were classified and measured in three ways. First an overall measure of the difference in performance between diabetic and non-diabetic groups across all cognitive abilities (general cognitive functioning) was calculated. The general cognitive functioning effect size is the average difference in scores between relevant groups across all cognitive abilities assessed across all studies. Second, differences between diabetic and non-diabetic groups on each cognitive ability were calculated (refer to Figure 2 for a list of all cognitive ability effect sizes calculated). Third, effect sizes for the differences between the diabetic and non-diabetic groups on neuropsychological measures that were used frequently enough across studies were calculated (refer to Figure 3 for a list of these tests).

To establish the classification of tests by the cognitive abilities measured, scores from each test were classified as belonging primarily to a specified cognitive ability. This was done according to Strauss, Sherman, and Spreen (2006) or Lezak and colleagues (2004) if the particular test score was not described in Strauss and colleagues. If a test was not found in Strauss et al. or Lezak et al. the classification from the study that used the measure was recorded and used to assign a cognitive ability to those measures. All measures used across studies included in the meta-analysis were assigned a cognitive ability with the reference page number supporting each choice documented. These classifications were then reviewed with a second subject matter expert (Dr. Anne Baird). Consensus on the classification of each test score according to the primary cognitive 
ability involved was reached, and all measures in the studies used in the meta-analysis ultimately were added to the master list shown in Appendix A. This approach was undertaken because there is not always an agreed upon or clear cut classification of a score from a given test.

Scores on the neuropsychological tests were classified as belonging to one the following cognitive abilities: focused attention, divided attention, selective attention, motor speed, processing speed/psychomotor efficiency, visuospatial/visuoconstructional, working memory, non-verbal reasoning, shifting, inhibition, semantic memory, verbal and visual learning and immediate recall, verbal and visual delayed recall, story learning and immediate recall, story delayed recall, verbal phonemic fluency, verbal semantic fluency, and design fluency (see Appendix A for a description of how these cognitive abilities were used to calculate effect sizes and which measures were included within each of them).

Coding for moderator variables. Study methodology was coded for sampling method used (population or case-control) and for design type (longitudinal or crosssectional). Publication year of each study or the year data was collected for longitudinal studies was recorded. Type of control group (non-diabetic or subset of diabetes patient group corresponding to one of the moderator variables) was coded along with inclusion and exclusion criteria. Age of the study participants was coded in years based on the mean (or median when available) age reported in individual studies. Sample size was recorded and used as a weight in the meta-analyses.

HbA1c level was coded based on the mean levels reported in each study. Fasting glucose level was coded based on the mean level reported in each study. Diabetes 
duration was coded as the number of years since diagnosis based on the mean number of years reported in each study. Insulin-dependent or non insulin-dependent diabetic status, presence of complications, diagnosis of depression or hypertension and publication year were coded as dichotomous variables (present or not present). Treatment modality for the insulin dependent/non-dependent variable, nature of complications (only studies that have measured complications with retinopathy or atherosclerosis were included), criteria or method for diagnosis of depression and hypertension and treatment status (i.e., whether treated or untreated for depression and hypertension), were recorded.

For the continuous theory driven moderator variables (age, HbA1c level, diabetes duration), in addition to studies that looked specifically at these variables, individual studies were coded based on the range of values of each of these variables in an individual study for their inclusion in the moderator variable meta-analyses.

Unfortunately, few studies reported the range of values or the range of values overlapped so that most studies could not be coded in this manner, as discussed in the results. See Appendix B for detailed coding criteria.

\section{Statistical Analyses}

Effect size calculation. Effect sizes (Cohen's $d$ ), representing the difference between the diabetic group and non-diabetic control group or the difference between subsets of diabetes patient groups in standard deviation units, were calculated for every score on the neuropsychological tests in individual studies. For the diabetic group and non-diabetic control group this was done for the scores that were classified by cognitive ability and on a test by test basis. For the moderator variable analyses this was done only for the scores that were classified by cognitive ability. 
To accomplish the first goal of the present study, average $d$ values were calculated for general cognitive functioning and each cognitive ability to determine the differences on these measures between the diabetic and the non-diabetic control groups. To accomplish the second goal of the present study, identifying the tests that are best able to measure cognitive deficits in T2DM, in instances where enough studies used the same neuropsychological test, the effect sizes for each of these tests were analysed to determine their ability to measure cognitive deficits in individuals with T2DM. These calculations were only done for the diabetes and non-diabetic control comparisons.

To accomplish the third goal, separate meta-analyses for cognitive deficits according to the hypothesized methodological moderator variables (sampling method, design, and publication year) were completed to compare subgroups of diabetic individuals on scores of classified cognitive abilities according to Hunter and Schmidt (2004, p.293). The calculations for these comparisons were the same as those completed for the diabetic and non-diabetic comparison (pp. 287-88). All other hypothesized moderator variable meta-analyses (diabetes duration, HbA1c levels, insulin-dependent or non insulin-dependent, presence of complications, and presence of depression or hypertension) could not be done due to lack of published studies looking at these moderator variables.

The individual effect size calculations were based on equations from Holmes (1984, equations 1, 18, and 21). Holmes uses the control group standard deviation for Cohen's $d$ calculation. The use of control group standard deviations is recommended when population data are used to calculate effect sizes (Hunter \& Schmidt, 2004). The present study used sample standard deviations as estimates of population standard 
deviations to calculate effect sizes. When sample standard deviations are used, it is recommend that a pooled standard deviation be calculated from the experimental and control group standard deviations, as this reduces error (Hunter \& Schmidt). Therefore in the present study, a pooled standard deviation weighted for sample size was calculated from the diabetes group and control group standard deviations, and this pooled standard deviation was used in all individual Cohen's $d$ calculations. The average effect size calculations weighted for sample size were based on equations from Hunter and Schmidt (pp. 287-88). Ninety-five percent confidence intervals were also reported. The number of studies and number of participants for each test score or cognitive ability classified was reported as not every study contained all tests or all cognitive abilities.

In meta-analyses comparing the diabetic group to non-diabetic controls, the diabetic group was the target group and negative effect sizes indicate that the diabetic group performed worse that the non-diabetic control group. For the moderator variable meta-analyses, the diabetes group of interest based on the moderator variable coded was the target group. Negative effect sizes indicate that the diabetic group of interest for a given coded moderator variable performed worse than the diabetic control group for that variable. A $p$ value equal or less than 0.05 indicated a significant difference between groups. Coding and calculations were done by the primary investigator (M.M.). Twelve of the twenty-four studies included in the meta-analysis were independently coded by a research assistant (R.A.). There were 16 initial differences between the coding of M.M. and the R.A. (13 omissions and three errors) out of 2220 units of information coded from the 12 studies. All differences in coding were resolved by consensus between M.M. and the R.A. and thus there was ultimately perfect inter-rater reliability. 
Statistic $\mathbf{Q}$ and fail-safe $\mathbf{N}$. The statistic $\mathrm{Q}$ was calculated to determine the heterogeneity of the effect sizes in the sample of studies used in this meta-analysis (Hedges \& Olkin, 1985; formula in Hunter \& Schmidt, 2004, p. 416). This statistic has been criticized for its low power (National Research Council, 1992); therefore a less conservative $p$ value of 0.10 was used. To rule out the possibility of publication bias influencing study results the fail-safe $\mathrm{N}$ was calculated to estimate the number of unpublished studies with null results needed to falsify any significant results that were found (Hunter \& Schmidt, pp. 500-1). Publication bias should not be a problem in this study as null findings are published in this area of research (Cosway et al., 2001). All analyses were calculated in Excel by M.M. according to equations in Hunter \& Schmidt and verified for accuracy using the StatsDirect statistical software package. 


\section{CHAPTER III}

\section{RESULTS}

\section{Article Inclusion in the Meta-Analysis}

Twenty-four articles were included in the meta-analysis comparing individuals with T2DM to non-diabetic controls. Figure 1 summarizes the search and exclusion process and Table 1 summarizes the characteristics of the twenty-four included studies, identified with an asterisk in the References. Two studies required the standard error of the mean to be converted to standard deviations to enable inclusion in the meta-analysis (Asimakopoulou, Hampson, \& Morrish, 2002; van Elderen et al., 2010).

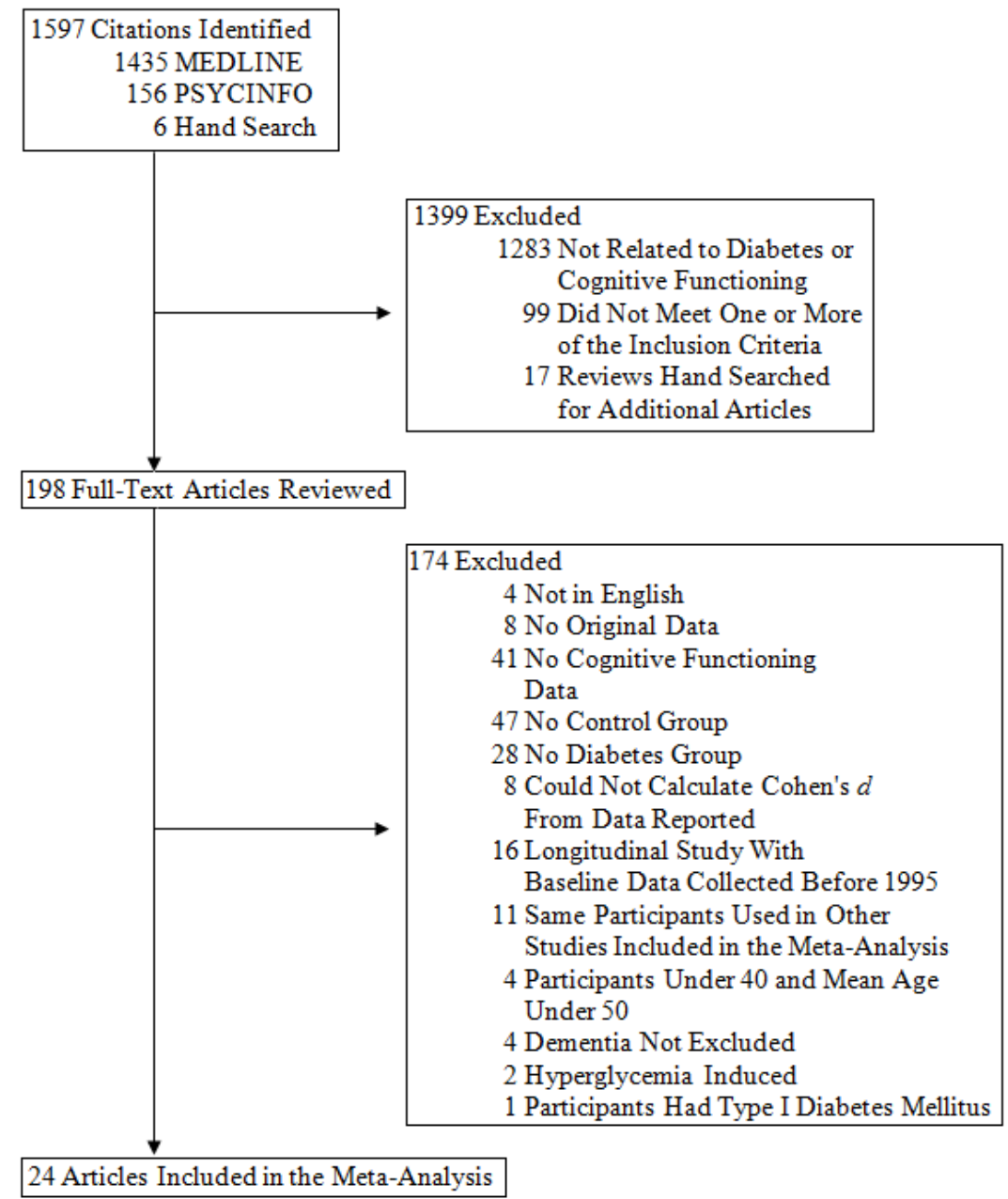

Figure 1 Flow Chart of Article Inclusion 
Table 1.

Characteristics of the Twenty-Four Studies Included in the Meta-Analysis

\begin{tabular}{|c|c|c|c|c|c|c|c|c|c|}
\hline $\begin{array}{l}\text { Lead author } \\
\text { and Year }\end{array}$ & $\begin{array}{c}\mathrm{n} \\
(\mathrm{DG} \\
/ \mathrm{CG}) \\
\end{array}$ & $\begin{array}{c}\text { Sex } \\
\text { (DG } \\
\text { /CG) } \\
\% \text { male } \\
\end{array}$ & $\begin{array}{c}\text { Age } \\
\text { (DG/CG) } \\
\text { Years } \\
\end{array}$ & $\begin{array}{c}\text { Education } \\
\text { (DG/CG) } \\
\text { Years } \\
\end{array}$ & $\begin{array}{l}\text { Study } \\
\text { design } \\
\text { code }\end{array}$ & $\begin{array}{c}\mathrm{HbA1c} \\
\% \mathrm{DG} \\
\end{array}$ & $\begin{array}{c}\text { Diabetes } \\
\text { duration } \\
\text { years DG }\end{array}$ & $\begin{array}{c}\text { Treatment } \\
\text { modality } \% \\
\text { for each } \\
\text { modality } \\
\text { DG }\end{array}$ & $\begin{array}{c}\text { Cohen's } d \\
\text { range (\# } \\
\text { contributing } \\
\text { to average } \\
\text { effect size } \\
\text { calculation) } \\
\end{array}$ \\
\hline $\begin{array}{l}\text { 1. Abbatecola } \\
\text { et al., } 2010^{\text {ad }}\end{array}$ & $\begin{array}{r}253 \\
1440\end{array}$ & N/A & $\begin{array}{r}76(7) \\
/ 74(6)\end{array}$ & $\begin{array}{r}6.3(3.3) \\
15.7(3.8)\end{array}$ & 4 & $\begin{array}{r}6.6 \\
(0.3)\end{array}$ & $9.8(5.9)$ & $\begin{array}{r}\text { D: 22; } \\
\text { O: 78; ITE }\end{array}$ & $\begin{array}{l}-0.25 \text { to } \\
0.07(5)\end{array}$ \\
\hline $\begin{array}{l}\text { 2. Arvanitakis } \\
\text { et al., 2004 }\end{array}$ & $\begin{array}{r}127 \\
/ 697\end{array}$ & $\begin{array}{r}44.9 \\
/ 28.7\end{array}$ & $\begin{array}{r}74.4(6.1) \\
/ 75.2(7.1)\end{array}$ & $\begin{array}{r}18.0(3.3) \\
/ 18.1(3.4)\end{array}$ & 2 & N/A & N/A & $\begin{array}{r}\text { D: } 33.1 \text {; } \\
\text { O: } 43.3 ; \\
\text { I: } 11.8 ; \\
\text { O+I: } 11.8\end{array}$ & $\begin{array}{r}-0.29 \text { to } \\
0.03(16)\end{array}$ \\
\hline $\begin{array}{l}3 . \\
\text { Asimakopoulou } \\
\text { et al., 2002 }\end{array}$ & 33 & 57.6 & $62.4(9.6)$ & N/A & 3 & N/A & $9(5.9)$ & $\begin{array}{r}\text { D: } 66.7 ; \\
\text { O: } 9.1 ; \\
\text { I: } 12.1 \\
\text { O+I: } 12.1\end{array}$ & $\begin{array}{l}-1.15 \text { to } \\
0.36(7)\end{array}$ \\
\hline $\begin{array}{l}\text { 4. Bent et al., } \\
2000\end{array}$ & $\begin{array}{r}168 \\
/ 2191\end{array}$ & $\begin{array}{r}56 / \\
\text { N/A }\end{array}$ & $\begin{array}{r}65.2(8) \\
/ 64.7(7.8)\end{array}$ & N/A & 3 & N/A & $\begin{array}{r}\mathrm{D}: \\
4.9(5.1) \text {; } \\
\mathrm{O}: \\
7.2(5.4) ; \\
\mathrm{I}: 17.6 \\
(14.1)\end{array}$ & $\begin{array}{r}\text { D: } 20.8 \text {; } \\
\text { O: } 59.5 \text {; } \\
\text { I: } 19.6\end{array}$ & $\begin{array}{r}-0.94 \text { to } \\
-0.85(2)\end{array}$ \\
\hline $\begin{array}{l}\text { 5. Bruehl et al., } \\
2007^{\mathrm{a}}\end{array}$ & 30 & 53.3 & $\begin{array}{r}59.2(8.6) \\
/ 59.1(8.4)\end{array}$ & $\begin{array}{r}15.5(2.4) \\
/ 16.2(1.9)\end{array}$ & 3 & $\begin{array}{r}7.5 \\
(1.5)\end{array}$ & 7.4(7.3) & $\begin{array}{r}\text { D: 26.7; } \\
\text { O: 73.3; } \\
\text { ITE }\end{array}$ & $\begin{array}{r}-1.08 \\
\text { to } 0.44(9)\end{array}$ \\
\hline $\begin{array}{l}\text { 6. Christman et } \\
\text { al., } 2010^{\mathrm{a}}\end{array}$ & $\begin{array}{r}28 \\
/ 150\end{array}$ & $\begin{array}{r}57.1 \\
148.7\end{array}$ & $\begin{array}{r}66.4 \\
(12.5) \\
/ 63.4 \\
(13.3)\end{array}$ & $\begin{array}{r}12.8(3.2) \\
/ 14.1(3.3)\end{array}$ & 1 & N/A & N/A & N/A & $\begin{array}{r}-0.76 \text { to } \\
-0.17(25)\end{array}$ \\
\hline $\begin{array}{l}\text { 7. Cosway et } \\
\text { al., } 2001^{\mathrm{a}}\end{array}$ & 37 & $\begin{array}{r}42.1 \\
139.5\end{array}$ & $\begin{array}{r}57.7 \\
(10.3) \\
/ 55.9 \\
(11.2)\end{array}$ & N/A & 3 & $\begin{array}{r}\text { Mdn: } \\
7.625^{\text {th }} \\
\text { \%ile: } \\
6.675^{\text {th }} \\
\text { \%ile: } \\
9.5\end{array}$ & $\begin{array}{r}\text { Mdn: } 6.0 \\
25 \text { th } \% \text { ile: } \\
3.0 \\
\text { 75th\%ile: } \\
11.3\end{array}$ & $\begin{array}{r}\text { D: } 21.1 ; \\
\text { O: } 52.6 ; \\
\text { O+I: } 10.5 ; \\
\text { I: } 15.8\end{array}$ & $\begin{array}{r}-0.33 \text { to } \\
0.19(11)\end{array}$ \\
\hline $\begin{array}{l}\text { 8. Espeland et } \\
\text { al., } 2011^{\text {ac }}\end{array}$ & $\begin{array}{r}179 \\
/ 1984\end{array}$ & 0 & $\begin{array}{c}65-59: \\
50.8 \% \\
146 \% \text {, } \\
70-74: \\
35.2 \% / \\
37.4 \% \text {, } \\
75-80: \\
14 \% / \\
16.6 \%\end{array}$ & $\begin{array}{r}\leq \mathrm{HS}: \\
28.5 \% / \\
26.4 \%,> \\
\text { HS: } 45.2 \% / \\
40.8 \%, \\
\text { CGr: } 26.3 \% \\
132.8\end{array}$ & 2 & N/A & N/A & N/A & $\begin{array}{r}-0.28 \text { to } \\
0.02(12)\end{array}$ \\
\hline $\begin{array}{l}\text { 9. Fuh et al., } \\
2007^{\mathrm{a}}\end{array}$ & $\begin{array}{r}72 \\
/ 144\end{array}$ & 0 & $\begin{array}{r}47.9(4.3) \\
\text { (R: 40- } \\
54)\end{array}$ & $3.3(3.8)$ & 1 & N/A & $4.9(5.7)$ & N/A & $\begin{array}{l}-0.51 \text { to } \\
0.03(6)\end{array}$ \\
\hline $\begin{array}{l}\text { 10. Gallacher et } \\
\text { al., } 2005\end{array}$ & $\begin{array}{r}165 \\
/ 1573\end{array}$ & 100 & $\begin{array}{r}61.7 \\
(\mathrm{R}: 55- \\
69)\end{array}$ & N/A & 1 & N/A & N/A & ITE & $\begin{array}{r}-0.19 \text { to } \\
-0.15(2)\end{array}$ \\
\hline $\begin{array}{l}\text { 11. Haroon et } \\
\text { al., } 2009^{\mathrm{b}}\end{array}$ & $\begin{array}{r}20 \\
/ 19\end{array}$ & $\begin{array}{r}35 \\
121\end{array}$ & $\begin{array}{r}57.7(7.9) \\
154.5(9.6)\end{array}$ & $\begin{array}{r}14.8(3.3) \\
/ 16.1(2.8)\end{array}$ & 3 & $\begin{array}{r}7.2 \\
(1.1)\end{array}$ & N/A & N/A & $\begin{array}{r}-0.34 \text { to } \\
-0.19(2)\end{array}$ \\
\hline $\begin{array}{l}\text { 12. Hewer et } \\
\text { al., } 2003\end{array}$ & $\begin{array}{r}53 \\
129\end{array}$ & N/A & R: $46-70$ & N/A & 3 & $\begin{array}{l}10.1 \\
(1.6)\end{array}$ & $12.0(6.4)$ & $\begin{array}{r}\text { O: } 60.4 ; \\
\text { I: } 15.1 ; \\
\text { O+I: } 24.5\end{array}$ & $\begin{array}{l}-0.69 \text { to } \\
-0.27(5)\end{array}$ \\
\hline
\end{tabular}




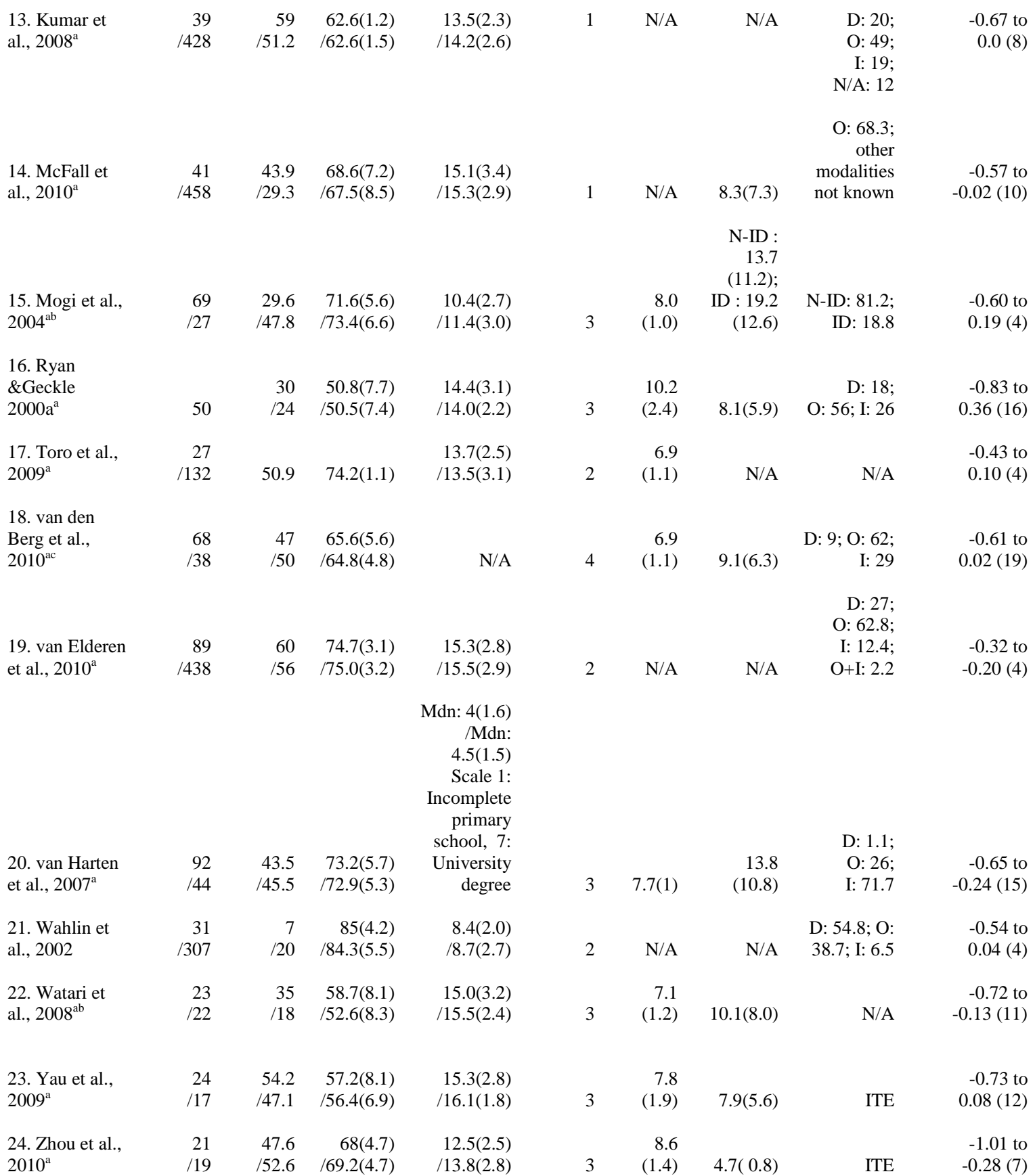

Note. All data are mean(SD) or \% unless otherwise specified. Data that are the same for the diabetes and control groups are only reported once. Mdn: median; R: range; \%ile: percentile; HS: high school; CGr: college graduate; DG: diabetes group; CG: control group; Study design code 1: population, cross-sectional; 2: population, longitudinal; 3: case-control, cross-sectional; 4: case-control, longitudinal; Treatment modality D: diet; O: oral hypoglycemic; I: insulin; O+I: oral hypoglycemic and insulin; ITE insulin treatment excluded; ID: insulin dependent; N-ID: non-insulin dependent. a studies that contributed scores to the test-by-test comparison

b studies that contained theoretical moderator variable analyses

c included in the 2000-2005 group for the publication year meta-analysis because data was collected prior to 2005

$\mathrm{d}$ not included in the publication year meta-analysis because data was collected from 2002-2009 


\section{Heterogeneity and Publication Bias}

The general cognitive functioning effect size was calculated in two ways, one in which individual Cohen's $d$ s were averaged within studies before the average general cognitive functioning effect size was calculated, and the other where individual Cohen's $d$ s were not averaged within studies before calculating the average general cognitive functioning effect size, to determine if there were differences between the two methods. The $Q$ statistic was significant for the diabetic versus non-diabetic comparison for general cognitive functioning when individual Cohen's $d$ s were averaged within studies so that each study in the meta-analysis only contributed one Cohen's $d$ to the general cognitive functioning effect size, $Q(23)=232.91, p<.10$. When the effect size for general cognitive functioning was calculated without averaging the Cohen's $d$ s within studies so that each study in the meta-analysis contributed more than one Cohen's $d$ to the general cognitive functioning effect size, the $Q$ statistic was reduced considerably but still remained significant, $Q(23)=119.14, p<.10$. The reduction in $Q$ indicates that there is less unexplained variance (or heterogeneity) in effect sizes across studies when individual Cohen's $d$ s are not averaged within studies compared to when they are averaged. Therefore, all subsequent effect sizes were calculated without averaging individual Cohen's $d$ s within studies.

The chosen method of effect-size calculation is not ideal as it has the potential to result in problems with independence of observations. It was chosen because of the reduction in $Q$ this method produced and it was also chosen to allow for the maximum number of effect sizes to be calculated. Had individual Cohen's $d$ s been averaged within studies, four of the cognitive ability effect sizes could not have been calculated and 
information on these four cognitive abilities would be lost. These four cognitive ability effect sizes could not have been calculated because there would not have been at least five individual Cohen's $d$ s making up these effect sizes and five is the minimum required number of individual Cohen's $d$ s for a reliable average effect size to be calculated.

The theory driven moderator variables analyses could not be completed due to a lack of published studies evaluating them. As a result, the Q statistic remained significant. Had it been possible to complete the theory driven moderator variable analyses further heterogeneity in effect sizes from the studies included in the metaanalysis might have been accounted for and this could have rendered the Q statistic nonsignificant.

The publication bias analysis (fail-safe $\mathrm{N}$ ) determined that 38 unpublished studies with null results would need to exist for the small general cognitive functioning effect size of -.26 to be reduced to a marginal value of -.10. It is not likely that 38 unpublished studies with null results exist in this area of research, as null results are often published in studies of cognitive functioning in T2DM. Furthermore, 14 of the 24 studies included in the meta-analysis had at least one null finding on measures of cognitive functioning (Asimakopoulou et al., 2002; Bruehl et al., 2007; Christman et al., 2010; Espeland et al., 2011; Fuh et al., 2007; Gallacher et al., 2005; Hewer et al., 2003; Kumar et al., 2008; McFall et al., 2010; Mogi et al., 2004; Toro et al., 2009; van Harten et al., 2007; Yau et al., 2009; Zhou et al., 2010) and four studies had only null findings (Abbatecola et al., 2010; Cosway et al., 2001; Haroon et al., 2009; Watari et al., 2008). 


\section{Diabetic Versus Non-Diabetic Controls: Comparison by Cognitive Abilities}

Average effect sizes with $95 \%$ confidence intervals by cognitive ability for the diabetic versus non diabetic comparison can be found in Figure 2. Every cognitive ability effect size was significant $(p<0.05)$ in the hypothesized direction, with those with T2DM performing worse on measures of all cognitive abilities than non-diabetic controls. The general cognitive functioning effect size was in the small range, $M=-.26,95 \% \mathrm{CI}[-.30$, .22]. Most cognitive ability effect sizes were in the small range. The focused attention, working memory, story learning and immediate recall, story delayed recall, and visuospatial/visuoconstructional ability effect sizes were in the marginal range. The cognitive abilities with the largest effect sizes were shifting/inhibition, $M=-.37,95 \% \mathrm{CI}$ $[-.48,-.27]$, which was in the moderate range and semantic memory, $M=-.67,95 \%$ CI [$.73,-.61]$, which was in the moderate to large range.

The shifting/inhibition average effect size was significantly larger than the motor speed average affect size $[t(1956)=12.99 p<0.001]$, which was the next highest effect size after the shifting/inhibition effect size. These two effect sizes differed by 0.07 standard deviation units. However, there was also a statistically significant difference between the motor speed and non-verbal reasoning average effect sizes $[t(4002)=2.68 p$ $<0.01]$, the next highest effect size after motor speed. These two effect sizes only differ by .01 standard deviation units. 


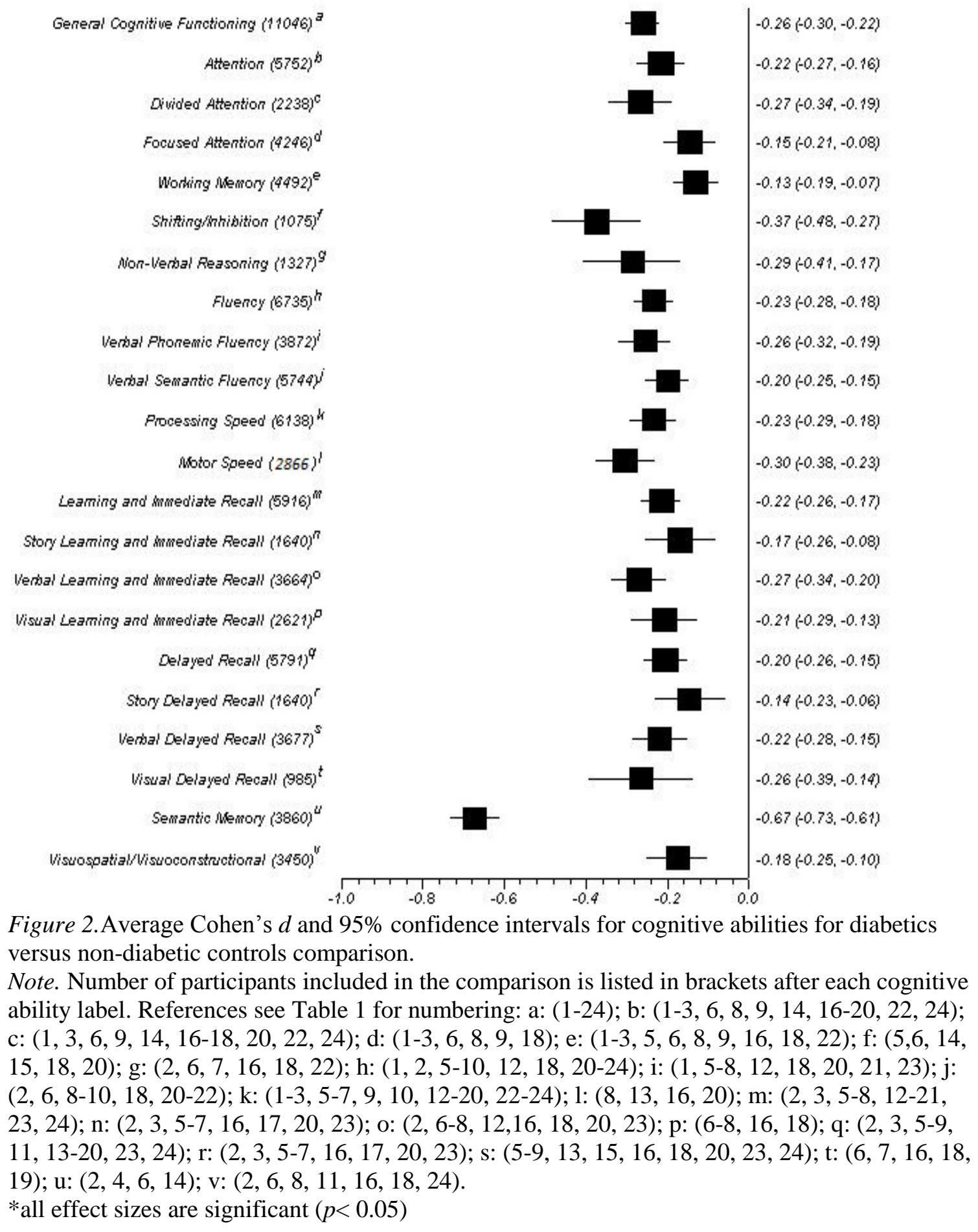




\section{Diabetic Versus Non-Diabetic Controls: Test-By-Test Meta-Analysis}

Average effect sizes with $95 \%$ confidence intervals by test for the diabetic versus non diabetic comparison can be found in Figure 3. Average effect sizes were calculated for every measure that was used in at least five studies. Every test effect size was significant $(p<0.5)$ in the hypothesized direction, with those with T2DM performing worse on all selected measures than non-diabetic controls. Most test effect sizes were in the marginal to small range. The tests with the largest effect sizes, which were all in the small to moderate range, were the Rey Auditory Verbal Learning Test (RAVLT) delayed recall $M=-.29,95 \%$ CI [-.46, -.13], Part III of the Stroop Colour-Word test, $M=-.33$, 95\% CI [-.44, -.22], and the Symbol Digit Modalities Test/WAIS-R Digit Symbol Substitution Test (SDMT), $M=-.46,95 \%$ CI [-.56, -.35]. There were significant differences between the RVALT delayed recall and Trail Making Test part B (TMT B) $t(1273)=7.61 p<0.001$ and the RVALT delayed recall and verbal fluency FAS $t(1119)$ $=7.82 p<0.001$, the two tests with the next largest effect sizes after the RAVLT delayed recall. The RAVLT delayed recall average effect size differed from the TMT B and FAS average effects size by 0.07 standard deviation units. 


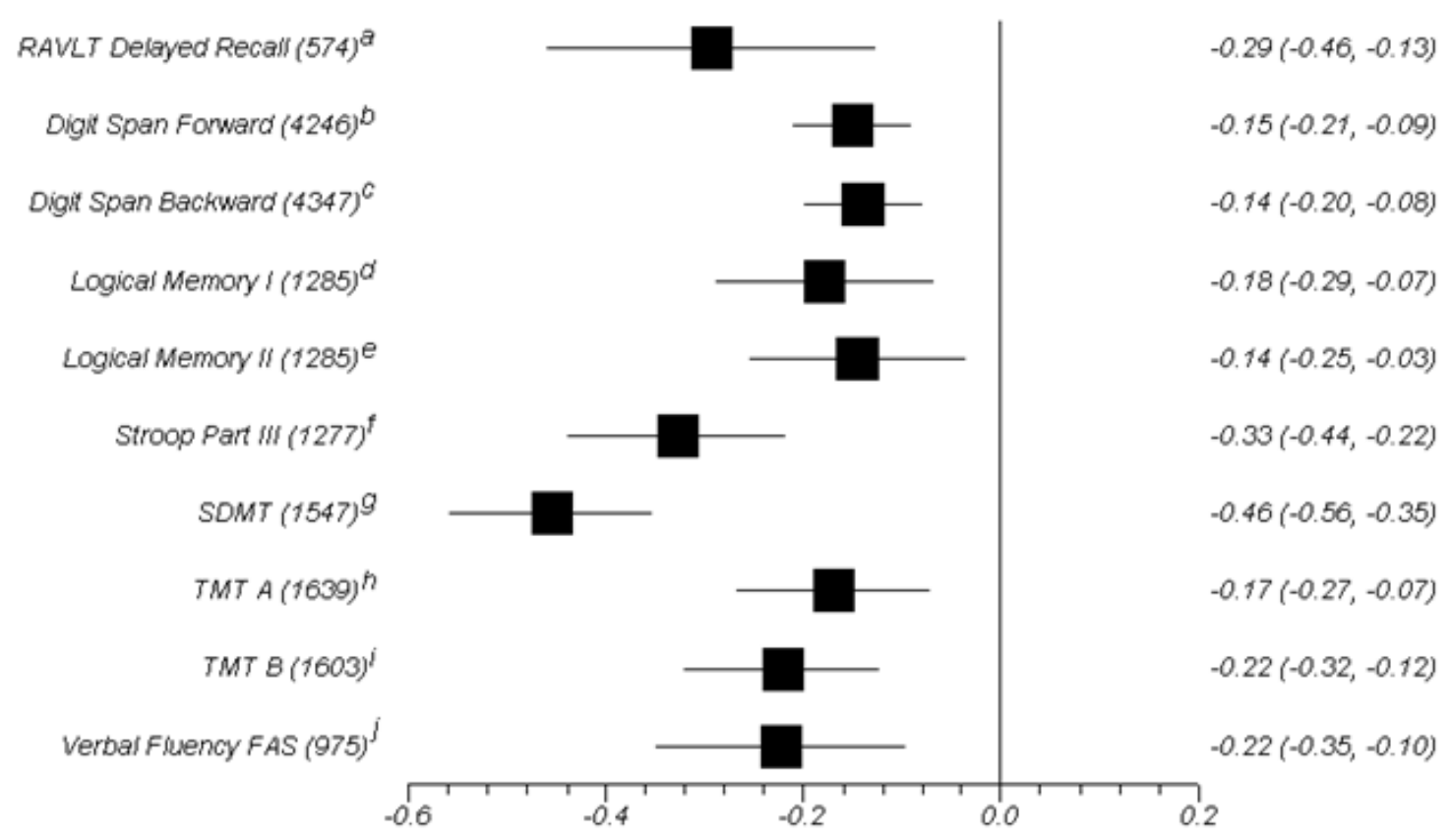

Figure 3.Average Cohen's $d$ and 95\% confidence intervals for selected neuropsychological measures for diabetics versus non-diabetic controls comparison.

Note. Number of participants included in the comparison is listed in brackets after each test label. RAVLT: Rey Auditory Verbal Learning Test; TMT: Trail Making Test; SDMT: Symbol Digit Modalities Test or the Digit Symbol Substitution Test from the WAIS-R; Digit Span Forward and Backward are from the WAIS-R, WAIS-III, or WMS-R; Logical Memory I\&II are from the WMS-R; References see Table 1 for numbering: a: $(7,9,18,20,24)$; b: $(1-3,6,8,9,18)$; c: $(1-3$, 5, 6, 8, 9, 18, 23); d: $(2,3,6,7,16,23)$; e: $(2,3,6,7,16,23)$; f: $(14,16,18,19,22)$; g: $(3,5,6$, 13-16, 23, 24); h: (1, 3, 6, 9, 17, 18, 20, 22, 24); i: (1, 3, 6, 9, 16-18, 22, 24); j: (1, 5, 20, 22, 23). *all effect sizes are significant $(p<0.05)$

\section{Moderator Variables Analyses}

There were not enough studies that met inclusion criteria looking at the proposed theoretical moderator variables for subgroup meta-analyses to be done. Table 2 shows individual Cohen's $d$ s for the three studies that looked at theoretical moderator variables.

The first study looked at depression, the second study looked at insulin dependent versus non-insulin dependent status, and the third study also looked at depression. The only significant difference from all three studies was a lower score on the WAIS-R Digit Symbol Substitution Test for those with insulin dependent T2DM when compared to those with non-insulin dependent T2DM and when compared to non-diabetic controls 
(Mogi et al. 2004). This significant difference is shown by two large range Cohen's $d$ s for the difference between those with insulin dependent T2DM and non-diabetic controls (1.29) and those with insulin dependent T2DM and those with non-insulin dependent T2DM (-.85). This study also found a moderate range effect size for the difference between those with non-insulin dependent T2DM and non-diabetic controls (-.46).

Only the Watari and colleagues (2008) study showed performance consistent with the hypotheses of the present study. In the Watari and colleagues study those with depression and T2DM performed worse than non-diabetic controls and worse than those with only T2DM on all measures. This finding supported the hypothesis that those with depression and T2DM would perform lower than those with only T2DM and also lower than controls. In addition, those with only T2DM performed worse than non-diabetic controls on all measures. 
Table 2.

Individual Cohen's ds for Three Theoretical Moderator Variable Studies

\begin{tabular}{|c|c|c|c|}
\hline Measure & \multicolumn{3}{|c|}{ Haroon et al. 2009 (Depression) } \\
\hline & DD vs. C & DD vs.NDD & NDD vs. C \\
\hline RCFT- copy & -0.12 & 0.17 & -0.34 \\
\hline RCFT- 3 minute delay & 0.00 & 0.20 & -0.19 \\
\hline RCFT- 30 minute recognition & -0.62 & -0.37 & -0.24 \\
\hline Measure & \multicolumn{3}{|c|}{ Mogi et al. 2004 (Treatment Modality) } \\
\hline & ID vs. $\mathrm{C}$ & ID vs. NID & NID vs. $\mathrm{C}$ \\
\hline Stroop interference score & -0.41 & 0.13 & -0.38 \\
\hline WAIS-R DSST & -1.29 & -0.85 & -0.46 \\
\hline ADAS word list immediate recall & -0.63 & -0.43 & -0.18 \\
\hline ADAS word list delayed recall & 0.06 & -0.19 & 0.23 \\
\hline Measure & \multicolumn{3}{|c|}{ Watari et al. 2008 (Depression) } \\
\hline & DD vs. C & DD vs.NDD & NDD vs. C \\
\hline Stroop Part I & -0.96 & -0.92 & -0.13 \\
\hline Stroop Part II & -0.72 & -0.54 & -0.17 \\
\hline Stroop Part III & -0.83 & -0.80 & -0.17 \\
\hline TMT A & -0.63 & -0.12 & -0.49 \\
\hline TMT B & -0.88 & -0.45 & -0.59 \\
\hline WAIS-III Digit Symbol Coding & -1.09 & -0.37 & -0.63 \\
\hline WAIS-III Letter-Number Sequencing & -0.78 & -0.01 & -0.72 \\
\hline WAIS-III Matrix Reasoning & -0.74 & -0.06 & -0.64 \\
\hline Verbal Fluency FAS & -0.84 & -0.18 & -0.59 \\
\hline Ruff Figural Fluency & -0.87 & -0.51 & -0.48 \\
\hline WCST category score & -0.46 & -0.35 & -0.13 \\
\hline
\end{tabular}

Note. Data are Cohen's $d$. Negative effect sizes reflect worse performance by the diabetes group with a moderator over the control group for the first comparison, worse performance by the diabetes group with a moderator over the diabetes only group for the second comparison, and worse performance by the diabetes only group over the control group for the third comparison. DD: depressed with diabetes; NDD: non-depressed with diabetes; ID: insulin dependent; NID: non-insulin dependent; C: controls; RCFT: ReyOsterrieth Complex Figure Test; WAIS-R DSST: Wechsler Adult Intelligence Scale Revised Digit Symbol Substitution Test; ADAS: Alzheimer's Disease Assessment Scale; TMT: Trail Making Test; WAIS-III: Wechsler Adult Intelligence Scale - Third Edition; WCST: Wisconsin Card Sorting Test.

Subgroup meta-analyses were performed for study sampling method, design, and publication year moderator variables. Average effect sizes for general cognitive functioning, calculated for the four possible combinations of study sampling method with study design, were as follows: population cross-sectional, $M=-.31,95 \% \mathrm{CI}[-.39,-.22]$; population longitudinal, $M=-.17,95 \% \mathrm{CI}[-.22,-.11]$; case-control cross-sectional $M=-$ 
$.57,95 \%$ CI [-.67, -..46]; and case-control longitudinal $M=-.15,95 \%$ CI [-.33, .03]. The largest average effect sizes came from case-control cross-sectional studies and the smallest from both longitudinal study designs. The average effect size for case-control longitudinal studies was not significant $(p>.05)$. A one-way analysis of variance for the difference among these four average effect sizes was significant $F(3,20)=4.522, p=$ 0.014. Calculating average effect sizes for the four possible study sampling method and study design combinations for general cognitive functioning further reduced the statistic Q value although it remained significant for all four study design effect sizes $(p<.10)$ and thereby suggests significant heterogeneity in effect sizes even within methodologically similar studies.

There were not enough studies to calculate cognitive ability effect sizes for the four possible combinations of study sampling method and study design. Therefore, further comparisons were made for cognitive abilities that had enough scores to calculate average effect sizes between case-control and population studies and between crosssectional and longitudinal studies as can be seen in Table 3. All comparisons were significant $(p<0.001)$ with case-control sampling methods showing significantly larger effect sizes than population sampling methods across all cognitive abilities with the exception of attention. Cross-sectional designs also had significantly larger effect sizes than longitudinal designs across all cognitive abilities. Also presented in Table 3 are the differences in average effect sizes for studies published between 2000 and 2005 and studies published between 2006 and 2012. Studies published between 2006 and 2012 had significantly larger effect sizes than studies published between 2000 and 2005 across all cognitive abilities. 
Table 3.

Study Design and Publication Year Meta-Analyses

\begin{tabular}{rrrr}
\hline Cognitive Ability & Moderator & Population & $t(d f)^{*}$ \\
\hline Attention & $-0.13(-0.23,-0.03)$ & $-0.26(-0.33,-0.19)$ & $-28.50(2077)$ \\
Fluency & $-0.30(-0.41,-0.18)$ & $-0.22(-0.28,-0.16)$ & $48.30(1525)$ \\
Working Memory & $-0.33(-0.45,-0.21)$ & $-0.08(-0.15,-0.01)$ & $20.14(1681)$ \\
Processing Speed & $-0.29(-0.40,-0.18)$ & $-0.21(-0.27,-0.15)$ & $17.77(2953)$ \\
L+I Recall & $-0.28(-0.42,-0.13)$ & $-0.21(-0.26,-0.16)$ & $8.85(788)$ \\
Delayed Recall & $-0.31(-0.45,-0.16)$ & $-0.19(-0.24,-0.13)$ & $13.80(850)$ \\
\hline & Cross-Sectional & Longitudinal & $t(d f)^{*}$ \\
\hline Attention & $-0.42(-0.53,-0.32)$ & $-0.16(-0.19,-0.04)$ & $140.19(2719)$ \\
Working Memory & $-0.33(-0.48,-0.18)$ & $-0.11(-0.16,-0.04)$ & $58.30(1374)$ \\
Fluency & $-0.30(-0.39,-0.22)$ & $-0.20(-0.26,-0.14)$ & $32.18(3066)$ \\
Processing Speed & $-0.31(-0.39,-0.24)$ & $-0.07(-0.17,0.03)$ & $88.89(5311)$ \\
L+ I Recall & $-0.24(-0.34,-0.14)$ & $-0.20(-0.26,-0.15)$ & $8.61(2093)$ \\
Delayed Recall & $-0.23(-0.33,-0.13)$ & $-0.19(-0.28,-0.13)$ & $7.69(2193)$ \\
\hline Attention & Published 2000-5 & Published 2006-12 & $t(d f)^{*}$ \\
\hline Fluency & $-0.13(-0.23,-0.04)$ & $-0.42(-0.53,-0.31)$ & $-84.57(3831)$ \\
Working Memory & $-0.09(-0.16,-0.02)$ & $-0.37(-0.54,-0.20)$ & $-66.52(921)$ \\
Processing Speed & $-0.17(-0.26,-0.16)$ & $-0.44(-0.58,-0.29)$ & $-35.41(728)$ \\
L+I Recall & $-0.20(-0.26,-0.14)$ & $-0.35(-0.43,-0.26)$ & $-59.60(5290)$ \\
Delayed Recall & $-0.17(-0.24,-0.10)$ & $-0.28(-0.38,-0.19)$ & $-24.68(4191)$ \\
\hline
\end{tabular}

Note. Data are Average Cohen's $d$ (95\% confidence interval). Welch's $t$ was used to correct for unequal group sizes and variances. L+I Recall: Learning and Immediate Recall

* all differences are significant $(p<0.001)$ 


\section{CHAPTER IV}

\section{DISCUSSION}

The present study sought to clarify and quantify the nature and pattern of cognitive deficits present in T2DM by comparing cognitive functioning in non-demented individuals with T2DM to that of non-demented and non-diabetic controls. This goal was addressed from three perspectives. First, it was addressed by looking at deficits in cognitive functioning between those with T2DM and non-diabetic controls on several cognitive abilities; these results are discussed in the cognitive ability analyses section. Second, it was examined by looking at deficits in cognitive functioning between those with T2DM and non-diabetic controls on neuropsychological measures used across studies; these results are discussed in the test-by-test analyses section. Lastly, it was met by looking at differences between subsets of the T2DM population on a number of methodological moderator variables; these results are discussed in the moderator variable analyses section.

\section{Cognitive Ability Analyses}

The present study found small significant effect sizes across most cognitive abilities when comparing those with T2DM to non-diabetic controls. There was some variation, with some effect sizes in the marginal range (focused attention, working memory, story learning and immediate recall, story delayed recall, and visuospatial/visuoconstructional), although these were still significant, and some effect sizes in the moderate range (shifting/inhibition and semantic memory).

Importantly, there was a statistically significant difference between the motor speed and non-verbal reasoning average effect sizes. These two effect sizes only differed by .01 standard deviation units, a difference that has no practical significance, and 
therefore this result is due to the large sample and subsequent high power that this comparison has. Therefore, any pattern in the deficits in cognitive abilities (i.e. reliably expected differences in performance on measures of cognitive abilities) should only be interpreted by the absolute magnitude of the effect size value (marginal, small, or moderate).

The semantic memory average effect size was the largest in the present study. This was unexpected as semantic memory is not a cognitive ability that is generally thought to be sensitive to changes from T2DM or age (Ryan \& Geckle, 2000b). Only six individual effect sizes from four studies were used to calculate the semantic memory average effect size (Arvanitakis et al., 2004; Bent et al., 2000; Christman et al., 2010; McFall et al., 2010). Two of these studies had participants who were matched on years of education and individual effect sizes from these two studies were marginal (.08 to .12, Arvanitakis et al.; McFall et al.). The study by Christman and colleagues was done in the United States and participants differed significantly on number of years of education and on ethnicity (a proxy for educational quality in the United States); the individual effect size from this study was moderate (.59). The last study by Bent and colleagues had the largest individual effect sizes (.85 and .94 , large range). This study did not report demographics for education or ethnicity and thus it is unknown whether these large effect sizes result from improperly matched groups on these demographic variables. Given the differences in individual effect sizes and inconsistent matching on number of years of education and educational quality, the semantic memory average effect size calculated in the present study is likely not a reliable measure of the influence of T2DM on cognitive functioning. 
Individual studies (as opposed to meta-analyses) in the literature had reported that the greatest deficits in cognitive functioning in T2DM were in verbal delayed memory and processing speed (Awad et al., 2004; Arvanitakis, et al., 2004; Biessels et al., 2008; Brands et al., 2007; Saczynski et al., 2008). In the present study, which combined results across studies, these two cognitive abilities had effect sizes in the small range and the divided attention, shifting/inhibition, non-verbal reasoning, verbal phonemic fluency, motor speed, verbal learning and immediate recall, visual delayed recall, and semantic memory cognitive ability effect sizes, which were all in the small to moderate range, were larger than the verbal delayed recall and processing speed cognitive ability effect sizes.

The findings from the present study, a meta-analysis of individuals with T2DM, were different from other meta-analyses investigating individuals with T1DM. Metaanalyses of children and adults with T1DM found no significant differences in learning and memory between those with T1DM and non-diabetic controls (Brands et al., 2005; Gaudieri et al., 2008). All learning and memory effect sizes in the present study showed significant differences between those with T2DM and non-diabetic controls. All learning and memory effect sizes in the present study were in the small range, with the exception of story learning and immediate recall and delayed recall effect sizes; these were in the marginal range. These differences in learning and memory deficits between those with T1DM and those with T2DM are likely due to an interaction of T2DM with age. Those with T1DM were children or adults under the age of 40 years and those with T2DM were for the most part adults older than 40 years of age. Learning and memory abilities decline with advancing age and T2DM represents a state of accelerated aging (Ryan \& Geckle, 
2000b). In addition, learning and memory abilities for contextual information such as stories decline more slowly with age than does memory for single words in a list, word pairs, or visual stimuli. Therefore marginal effect sizes for story learning and immediate recall and delayed recall are not unexpected (Ryan \& Geckle, 2000b).

Van den Berg and colleagues (2009) reported median effect sizes for a small subset of the studies included in their review. They reported median effect sizes for processing speed (Cohen's $d=-.40$ ), attention (Cohen's $d=-.50$ ), and memory (Cohen's $d=-.30$ ) as broad cognitive domains. Comparable median effect sizes from the present study are (Cohen's $d=-.40$ ) for processing speed (Cohen's $d=-.33$ ) for attention, and (Cohen's $d=-.28$ ) for memory.

\section{Test-By-Test Analyses}

Few neuropsychological measures were used often enough across studies to permit effect size calculation. All measures for which effect sizes could be calculated had significant effect sizes ranging from marginal to moderate in size. Although the cognitive ability analyses did not support the hypothesis that delayed verbal memory and processing speed would have the largest effect sizes, the test-by-test analyses did support this hypothesis. The RAVLT delayed recall score is a measure of delayed verbal memory and the SDMT is a measure of processing speed. These were the tests with the largest effect sizes along with a selective attention test part III of the Stroop Colour-Word Test. All three tests had effect sizes in the moderate range.

Since those with T2DM do not usually begin to show cognitive deficits until later in life and because the cognitive deficits in T2DM have been characterized as accelerated aging (Biessels et al., 2008; Okereke et al., 2008), neuropsychological measures that are 
sensitive to early cognitive deficits in aging likely also would be sensitive to early cognitive deficits in T2DM. As such, measures that could form the basis for baseline assessments in older adults with T2DM include: the Wechsler Memory Scale - Fourth Edition, the Trail Making Test A \& B, the Clock Drawing Test, the Wechsler Abbreviated Scale of Intelligence, the Boston Naming Test, the Controlled Oral Word Association Test and Animal Fluency, and the Wisconsin Card Sorting Test (Rabin, Wishart, Fields, \& Saykin, 2006). The Symbol Digit Modalities Test, the Stroop ColourWord Test, and the Rey Auditory Verbal Learning Test should also be included based on the larger effect sizes for these measures found in the present study. Use of these tests should be considered in research studies and in clinical settings as measures which may be sensitive to incipient cognitive dysfunction in older adults with T2DM who are suspected of showing cognitive decline.

\section{Moderator Variables Analyses}

Only three studies met inclusion criteria for theoretical moderator variable analyses, two that examined depression and one that examined treatment modality (Haroon et al., 2009; Mogi et al., 2004; Watari et al., 2008) and only one of these studies showed the hypothesized results (Watari et al.), with those with the moderator variable (depression) and T2DM performing worse than those with T2DM alone and those with T2DM alone performing worse than non-diabetic controls. Had it been possible to conduct the subgroup meta-analyses the statistic $Q$ would likely have become nonsignificant as additional unexplained variance (heterogeneity in effect sizes) would have been accounted for by the theoretical moderator variable analyses. 
Performing separate meta-analyses by study sampling method and study design showed significant differences in selected cognitive ability effect sizes. Case-control cross-sectional studies had the largest effect sizes, followed by population cross-sectional studies. Case-control longitudinal and population longitudinal studies had the smallest effect sizes. For all longitudinal studies included in the meta-analysis, only baseline data was used in effect size calculation to maintain consistency across studies. Because baseline data were used, individuals with T2DM in these studies were younger, and were selected to be healthier at baseline than individuals in cross-sectional studies that only measured cognitive functioning at one point in time. This likely resulted in smaller effect sizes for cognitive functioning deficits from longitudinal studies than from crosssectional studies. Similarly, population studies that recruited participants from community samples had smaller effect sizes than case-control studies that recruited individuals from in-patient and out-patient treatment settings. Individuals from community samples likely were healthier overall than individuals from in-patient or outpatient settings even though both groups had T2DM. Overall, longitudinal design had the greatest influence on effect sizes as shown by the marginal effect sizes for both casecontrol and population longitudinal studies.

Studies published between 2006 and 2012 had significantly larger effect sizes than studies published between 2000 and 2005. It was predicted that since T2DM management has improved dramatically in recent years newer studies would show smaller effect sizes. This was not the case. Although it is likely that participants with diabetes in recent studies have better managed T2DM, twelve years may not be a long enough time span for this improvement to be reflected in cognitive ability effect sizes. 
Older studies would need to have been included in the meta-analysis to show this effect. The significant difference in effect sizes favouring studies published between 2006 and 2012 is likely an artifact of the distribution of studies with different sampling methods and designs. Studies published between 2000 and 2005 contained four longitudinal studies and seven cross-sectional studies, while studies published between 2006 and 2012 contained one longitudinal study and 11 cross-sectional studies. Given the smaller effect sizes from the longitudinal studies and the larger effect sizes from the cross-sectional studies, the average effect sizes for studies published between 2000 and 2005 were probably pulled down by the effect sizes contributed by the longitudinal studies, and likewise the average effect sizes for studies published between 2006 and 2012 were probably pulled up by the effect sizes contributed by the cross-sectional studies.

\section{Limitations}

The cognitive ability effect sizes that were calculated in the present study were dependent upon enough individual studies including measures of the given ability. Some of the cognitive ability effect sizes calculated had relatively few scores included (motor speed, semantic memory, visual learning and immediate recall, and visual delayed recall) and some cognitive abilities had to be combined in order to calculate an effect size for them (inhibition and shifting were combined, selective attention was only calculated as part of attention, and design fluency was only calculated as part of fluency). These restrictions on calculated effect sizes would have decreased the reliability of the effect sizes calculated with fewer scores and as summary abilities.

Similarly, few individual studies used the same neuropsychological measures, and therefore there was a restricted number of measures for which average effect sizes could 
be calculated in the test-by-test meta-analyses. Lastly, subgroup meta-analyses for the theoretical moderator variables could not be done because very few studies looked at the effects of moderator variables using separate groups. This resulted in significant levels of unexplained heterogeneity remaining in the present study.

\section{Future Research}

The statistic $Q$ indicated significant heterogeneity among effect sizes within the same set of analyses. This heterogeneity likely occurred because theoretical moderator variable subgroup meta-analyses could not be calculated with the available published studies. Future research should report data in a way that would allow for subgroup metaanalyses or meta-regression to be done. Without subgroup meta-analyses or metaregression it will not be possible to know across studies which moderator variables have the greatest statistical impact and which ones are most likely to increase the risk for cognitive dysfunction in T2DM. Individual studies have begun to look at mediation and moderation models of theoretical moderator variables using multiple regression analysis and structural equation modeling (for example McFall et al., 2010). However, individual models produced in these studies cannot be consolidated across studies to perform metaregressions.

Subgroup meta-analyses will accomplish the goal of quantifying the impact of individual moderator variables on cognitive functioning in T2DM across studies, which has yet to be done. Meta-regression will be able to evaluate multiple moderator variables together, provided certain conditions are met within individual studies (Baker, White, Cappelleri, Kluger, \& Coleman, 2009). First, means and standard deviations must be reported for the diabetes group and for the control group or for the diabetes group with a 
given moderator variable and for the diabetes group with only diabetes. Without means and standard deviations, or at the very least $t$ or $F$ statistic values for mean comparisons, data from individual studies cannot be compared across studies. Second, moderator variables must be reported in the same metric across studies to allow meta-regression to be performed because all values across studies need to have the same meaning to be comparable across studies. These could be means and standard deviations in years for age and duration of illness, means and standard deviations of $\mathrm{HbAlc}$ level, percentage or $n$ of participants receiving each treatment modality, and the percentage of the sample with a specified comorbid condition or diabetes complication. Third, because meta-regression only allows for one dependent variable, neuropsychological measures across studies need to include measures of the same cognitive abilities across studies to enable calculation of a summary cognitive functioning score that encompasses these same cognitive abilities. Lastly, each moderator variable included in a meta-regression requires a minimum of ten studies. Therefore there is a need for many more studies evaluating cognitive functioning in T2DM for a meta-regression encompassing multiple moderator variables to be performed.

Individual studies of cognitive functioning in T2DM should strive to consistently measure a broad sampling of the same cognitive abilities using the same neuropsychological measures. A conference could be held with neuropsychologists and diabetes educators who specialize in working with those with T2DM to determine what cognitive abilities these should be and which tests should be used to measure them.

With clinical goals in mind, most moderator variables can be conceptualized as a progression to a more severe disease state. Higher HbA1c levels, insulin treatment, and 
diabetes complications are directly related to treatment adherence and disease management and comorbid conditions such as hypertension and depression indirectly affect treatment adherence and disease management by complicating it. Poor and complicated diabetes management significantly increases the risk for cognitive deficits and dementia (Awad et al., 2004). If treatment adherence and diabetes management could be improved in older adults, this could minimize the risk of cognitive deficits and dementia in this population. Diabetes management could be simplified and improved by individually tailoring treatment strategies or interventions that would optimize treatment adherence to cognitive strengths and weaknesses (Monette, 2012). As those with T2DM age, the role of neuropsychology in diabetes treatment management should significantly increase through assessments of cognitive functioning in those at risk of cognitive decline and through individually tailoring treatment regimens to cognitive strengths and weaknesses.

\section{Conclusion}

The present study found statistically significant small deficits across all measures of cognitive abilities in individuals with T2DM when compared to non-diabetic controls. In individual studies, sampling method and study design affected results, case-control studies yielded larger effect sizes than population studies and cross-sectional studies had larger effect sizes than longitudinal studies, in which baseline measurements were used for calculation of effect sizes. The high number of null findings in individual studies suggests that when cognitive functioning deficits are present in T2DM, they are larger in magnitude than the small average effect sizes reported in this study. Null-findings in the literature mask the magnitude of cognitive functioning deficits when they are present 
because data are averaged across studies. Individuals with T2DM who are seen clinically by neuropsychologists will likely have cognitive functioning deficits that are greater in magnitude than the small effect sizes reported in the present study. This is because individuals with T2DM who present for clinical neuropsychological assessment have typically noticed or exhibited some change in cognitive functioning, thus they are likely to be a more impaired subset of the overall population of individuals with T2DM. This should be a consideration when clinical work is done with individuals who have T2DM. 


\section{APPENDICES}

\section{APPENDIX A}

Glossary of Cognitive Abilities with Classification

For the purposes of effect size calculation, all test scores from individual studies were assigned to the primary cognitive ability the test score measured. All classifications were based on the cognitive abilities that Strauss, Sherman, and Spreen (2006) and Lezak, Howieson, and Loring (2004) state each test score measures. Strauss and colleagues was consulted first. If a test was not discussed in Strauss and colleagues, Lezak and colleagues was then consulted. If a test score was not found in Strauss et al. or Lezak et al., the cognitive ability classification for that test score came from the individual study that used the test score. The cognitive ability classification was consolidated in order to maximize the number of effect sizes to be calculated by assigning the same cognitive ability to as many tests as possible. If a classification was different from what was in Strauss, Lezak, or an individual study, this is mentioned and a rationale/justification for changing the classification is provided. Any differences in administration, task demands, and scoring of tests within a particular cognitive ability are accounted for by calculating individual Cohen's $d$ s for each score from each study, which standardizes the scores from individual studies. These individual Cohen's $d$ s are then used to calculate average Cohen's $d$ s across all studies for each cognitive ability.

General Cognitive Functioning encompasses all cognitive abilities grouped together and is a summary average effect size of all neuropsychological test scores across all studies. 
Motor Speed includes motor coordination, dexterity, and speed.

Test scores for motor speed. The Purdue Pegboard Test, the Grooved Pegboard Test, and the Finger Tapping Test were included. Raw scores from the Purdue Pegboard Test are the number of pegs placed in 30 seconds, with the dominant hand, the nondominant hand, and both hands. Grooved Pegboard Test scores are the time needed to insert 25 pegs with the dominant and with the non-dominant hands. Finger Tapping Test scores are based on the mean number of taps completed over several 10 second trials with the dominant and non-dominant hands.

Processing Speed/Psychomotor Efficiency is the ability to perform relatively easy or over-learned cognitive tasks and the ability to process information automatically and fluently.

Test scores for processing speed/psychomotor efficiency. All processing speed tasks require some sort of repetitive easy or over-learned response to be completed in a given time or measured by the total time needed to complete the task. Some tasks involve using a novel alphanumeric code and completing as many items as possible in a given time (90-120 seconds) either with a motor or oral response (WAIS-R Digit Symbol Substitution subtest, Symbol Digit Modalities Test: paper-pencil or oral, WAIS-III Digit Symbol Coding, Letter-Digit Coding Test). Some tasks involve identifying various target stimuli from a number of distractors as quickly as possible (Number Comparison test, Cancellation tasks, Salthouse Perceptual Comparison Test, Embedded Figures Test, Digit Vigilance test). The Trail Making Test part A measures the total time needed to sequence randomly dispersed numbers. The Stroop Colour-Word test Part I measures speed with 
which colour words are read when presented in black ink. The Stroop Colour-Word test Part II measures speed with which colours are named from colour name words printed in an ink colour congruent with the colour name. Simple, Two Choice, or Four Choice Reaction Time measures with scores reported as mean latency time in seconds or millisecond also measure processing speed.

Visuospatial/Visuoconstructional is the ability to perceive, organize, and construct visuospatial stimuli. Although visuospatial and visuoconstructional abilities are often seen as separate, with visuospatial more closely related to perception and visuoconstructional more closely related to construction/manipulation, they are related abilities and have been included together as a broader cognitive ability for the purposes of effect size calculation. If these abilities were not combined there would not have been enough scores for either ability to calculate an effect size.

Test scores for visuospatial/visuoconstructional. The copy trials of the ReyOsterrieth Complex Figure Test and the Taylor Complex Figure Test require the accurate reproduction of a complex abstract geometric figure with no time constraint. The score is the number of accurately copied details from the figure. Block Design from the WAIS-R requires the reproduction of pictured designs using blocks in a given time; the score is the number of accurately reproduced designs in a given time. The Clock Drawing Test requires drawing or copying the face of a clock and placing the numbers, hands, and a given time on the clock face. The score is the number of correctly placed details on the clock face. The Judgment of Line Orientation test requires the matching of partial line segments representing angles of various sizes to a stimulus picture. The score is the 
number of line segments correctly matched to the stimulus picture. The Card Rotations Test is a mental rotation task; the score is the number of figures correctly matched to the same figure presented in a different rotation in space. The Object Assembly subtest of the WAIS-R measures the ability to put together representations of known objects in a given time.

Attention includes divided, selective, and focused attention. Individual effect sizes were calculated for divided attention and for focused attention. A summary attention effect size was calculated that included the scores from the divided attention and focused attention measures as well as measures of selective attention. There were not enough measures of selective attention for this ability to have an individual effect size.

Divided attention. Divided attention is the ability to perform multiple tasks or multiple task demands simultaneously.

Test scores for divided attention. The Brief Test of Attention requires listening to a string of numbers and letters and identifying how many numbers are presented while ignoring the letters in one instance and how many letters are presented while ignoring the numbers in the other. The score is the number of correctly completed trials (max. score 10) for each of the two conditions. The Trail Making Test part B is a paper and pencil alternating sequencing task involving numerals and letters that are randomly dispersed on a page. The score is completion time. The Color Trails Test part 2 is the same as the Trail Making Test part B, except all the numbers are encircled by the same colour and all the letters are encircled by the same colour that is a different colour from the one used for the numbers. The score is time to completion. The difference in time between the Trail 
Making Test part A and part B or a ratio of these two scores are also considered to be measures of divided attention.

Focused attention. Focused attention is the ability to concentrate attention to relevant stimuli to perform a specific task

Test scores for focused attention. The tests of focused attention included require the repetition of a string of digits of increasing length (WAIS-R, WMS-R, and WAIS-III Digit Span Forward) or a sequence of tapped blocks of increasing length in an array (Corsi Block-Tapping Test Forward).The Corsi Block-Tapping Test Forward is assigned to the ability of attentional capacity by Lezak and colleagues (2004), it has been included here under focused attention as a more specific classification because of its similarity to the digit span tests. The scores are either the number of items completed or the longest string of digits or block sequences completed.

Selective attention. Selective attention is the ability to continue a response despite distracting or competing stimuli, referred to as "freedom from distractibility." Test scores for selective attention. The only test of selective attention, the Stroop ColorWord test Part III, is scored as the time needed to name the colour of ink for a series of colour name words, in which the colour of the ink and the colour name words are not congruent. There are a given number of these mismatched colour-word pairs printed on a card.

Inhibition/Shifting the ability to inhibit well learned responses and inappropriate responses according to task demands and the ability to switch responses and respond flexibly according to task demands. Inhibition and shifting were combined into one effect 
size because they are both components of executive functioning and because there were not enough test scores to calculate individual inhibition and shifting effect sizes.

\section{Test scores for inhibition and shifting.}

Inhibition. The Hayling Sentence Completion test requires completing sentences with words that logically complete the sentence and with words that are unrelated to the sentence. Larger differences in time to complete both tasks represent poorer inhibition. The difference in time between Part III and Part II of the Stroop Colour-Word Test is a measure of inhibition.

Shifting. The Brixton Spatial Anticipation test, the Wisconsin Card Sorting Test, and the modified Wisconsin Card Sorting Test all require the ability to determine a rule for sequencing or sorting stimuli. All three tests have an error score that represents difficulties with shifting.

Non-Verbal Reasoning is the ability to understand non-verbal concepts and problemsolving using non-verbal means.

Test scores for non-verbal reasoning. The Picture Completion subtest from the WAIS-R requires identifying the missing portion of an image. The score is number of correctly completed items. The Matrix Reasoning subtest of the WAIS-III, The Raven Advanced Progressive Matrices, and the Standard Progressive Matrices require choosing the correct image from an array of possible choices to complete a pattern in a pictured matrix, the score is the number of correct items. Matrix Reasoning, although classified as fluid reasoning in Strauss and colleagues (2006), has the same task demands as the Raven Progressive Matrices test, which is classified as non-verbal reasoning in Strauss and 
colleagues (2006).Therefore, Matrix Reasoning has been classified under non-verbal reasoning for the purposes of effect size calculation. The Tactual Performance Test requires placing ten shapes in their corresponding location on a form board while blindfolded. The score is total time for completion with the dominant hand, the nondominant hand, and with both hands. The Category Test requires deducing an underlying organizing principle for presented stimuli by making subsequent responses that incorporate feedback. The score is the number of errors made over all subtests. The Wisconsin Card Sorting Test and the modified Wisconsin Card Sorting Test require sorting cards into six categories based on the shape, number, and colour of items presented on the cards in response to feedback. The score is the number of correctly sorted categories when sorting 128 cards.

Working memory is the ability to retain information in the short-term, and to manipulate and work with that information.

Test scores for working memory. Backward span tests require the repetition of a string of digits of increasing length in the reverse order in which they are presented (WAIS-R, WMS-R, and WAIS-III Digit Span Backward), or a sequence of tapped blocks of increasing length in an array (Corsi Block-Tapping Test Backward). The scores are either the number of items completed or the longest string of digits or block sequences completed. Ordering tasks require putting an increasing number of presented numbers (Digit Ordering Test), letters (Alpha Span), or letters and numbers (Letter-Number Sequencing from WAIS-III) in order, where the scores are the number of items completed. The Four-Word Short-Term Memory test requires remembering four 
presented words after counting backwards for 5, 15, or 30seconds. The score is the number of correctly remembered words over 15 trials.

Fluency includes design, verbal phonemic, and verbal semantic fluency. Individual effect sizes were calculated for verbal phonemic fluency and for verbal semantic fluency. A summary fluency effect size was calculated that includes the scores from the verbal phonemic fluency and verbal semantic fluency measures as well as measures of design fluency. There were not enough measures of design fluency for this ability to have an individual effect size.

Verbal phonemic fluency. Verbal phonemic fluency is the ability to generate words that begin with a given letter in a given time.

Test scores for verbal phonemic fluency. All tests require naming as many words as possible that begin with a certain letter in one minute. Scores are either the mean number of words produced for two or three letters or the total number of words produced for all letters given. Possible letters are $\mathrm{N}+\mathrm{A}, \mathrm{F}+\mathrm{A}+\mathrm{S}, \mathrm{S}+\mathrm{F}, \mathrm{N}+\mathrm{S}$, and $\mathrm{J}+\mathrm{S}+\mathrm{M}+\mathrm{U}$.

Verbal semantic fluency. Verbal semantic fluency is the ability to generate words that belong to a particular semantic category in a given time

Test scores for verbal semantic fluency. All tests require naming as many words as possible that belong to a certain semantic category in one minute. Scores are the total number of words produced for all categories given. Possible categories are animals, fruits and vegetables, occupations, and grocery store items.

Design fluency. Design fluency is the ability to generate simple geometric designs in a given time. 
Test scores for design fluency. All tests require producing as many unique designs as possible in one minute. Scores are the total number of unique designs produced.

Memory Measures The memory measures differ on a number of dimensions, specifically by the stimuli used (single words, word pairs, stories/paragraphs, visual stimuli), the number of learning trials, the number of items to be learned, the recall paradigm (incidental or intentional, free or cued), and the length of delay for delayed recall (ranges from 3 to 30 minutes). Separate effect sizes for story learning and immediate recall, multiple learning trial verbal learning and immediate recall, and multiple learning trial visual learning immediate recall were calculated as there were enough test scores across studies to do this. A summary learning and immediate recall effect size was also calculated including these three abilities and others as described below.

Separate effect sizes for story delayed recall, verbal delayed (free) recall, and visual delayed recall, each with 15 to 30 minute delays, were calculated as there were enough test scores across studies to do this. A summary delayed recall effect size was also calculated including these three abilities and others as described below. Summary immediate recall and delayed recall effect sizes provide clinically important information, despite encompassing tasks with different administrations and materials. Although story memory tasks are also part of verbal memory, a separate effect size was calculated for these because recall of organized contextual information such as stories represents a 
different cognitive ability than memory for lists of single words or learned associations between word pairs. A semantic memory effect size was also calculated.

Verbal Learning and Immediate Recall is the ability to learn and recall verbal information over multiple trials immediately after stimuli presentation

Test scores for verbal learning and immediate recall. Scores are the total number of words recalled from word lists immediately after 3-6 learning trials and include the Rey Auditory Verbal Learning Test, the Hopkins Verbal Learning Test Revised, and the word list from the Consortium to Establish a Registry for Alzheimer's Disease. There are also scores for immediate recall from two tests where it is required to learn associations between pairs of unrelated words over multiple trials, the Verbal Pairs I subtest from the WMS-R and the Verbal Paired-Associates Learning Test.

Verbal Delayed Recall is the ability to recall verbal information after a delay ranging from 15 to 30 minutes.

Test scores for verbal delayed recall. Scores are the total number of words recalled from word lists after a 15 to 30 minutes delay and include the Rey Auditory Verbal Learning Test, the Hopkins Verbal Learning Test - Revised, and the California Verbal Learning Test. There are also scores for 30 minutes delayed recall for the Verbal Pairs II subtest from the WMS-R and the Verbal Paired-Associates Learning Test. Story Learning and Immediate Recall is the ability to learn and recall information from a story or paragraph with a logical structure immediately after story presentation.

Test scores for story learning and immediate recall. All story recall tasks included require the immediate recall of the story or paragraph immediately after it has been read, all scores are based on the number of details correctly recalled. Story recall 
tests include Logical Memory I from WMS-R, the story from the East Boston Memory Test, the paragraph from the Guild test, the story from the Rivermead Behavioural Memory Test, and the neutral paragraph from the Emotional Memory Test.

Story Delayed Recall is the ability to recall information from a story or paragraph with a logical structure after a delay ranging from 15 to 30 minutes.

Test scores for story delayed recall. All story recall tasks included require the delayed recall of the story or paragraph ranging from 15 to 30 minutes after it has been read, all scores are based on the number of details correctly recalled. Story recall tests include Logical Memory II from WMS-R, the story from the East Boston Memory Test, the paragraph from the Guild test, the story from the Rivermead Behavioural Memory Test, and the neutral paragraph from the Emotional Memory Test.

Visual Learning and Immediate Recall is the ability to learn and recall visual stimuli over multiple trials immediately after stimuli presentation.

Test scores for visual learning and immediate recall. All tests included require the immediate recall after 3-5 trials of presented stimuli, which include geometric figures (Visual Reproduction I, and Visual Pairs I from the WMS-R, the Brief Visuospatial Memory Test-Revised, and the Benton Visual Retention Test), locations (Location Learning Test), and associations between symbols and digits (Symbol-Digit PairedAssociate Learning Test).

Visual Delayed Recall is the ability to recall visual stimuli after a delay ranging from 15 to 30 minutes.

Test scores for visual delayed recall. All tests included require the delayed recall of presented stimuli, which include geometric figures (Visual Reproduction II, and Visual 
Pairs II from the WMS-R, the Brief Visuospatial Memory Test-Revised, and the Taylor Complex Figure Test), associations between symbols and digits (Symbol-Digit PairedAssociate Learning Test), and pictures (Picture Learning Test).

Learning and Immediate Recall is the ability to recall presented stimuli after no delay. Test scores for learning and immediate recall. The tests included under the Verbal learning and immediate recall, Story learning and immediate recall, and Visual learning and immediate recall cognitive abilities were included under the Immediate recall ability. Additional tests included measures of verbal single trial immediate free recall: total number of words recalled on Trial 1 of List A and List B from the Rey Auditory Verbal Learning Test (max. score 15), the total number of words recalled after one learning trial for the Word list from the Alzheimer's Disease Assessment Scale (max. score 10), and from a task involving free recall of random words (max. score 12), measures of verbal single trial immediate cued recall: the total number of words recalled after one learning trial when presented with a semantic cue from a task involving cued recall of a list of organisable words (max. score 12), and measures of visual single trial immediate recall: Picture Learning Test, total number of pictures recalled immediately after stimulus presentation.

Delayed Recall is the ability to recall presented information after a delay ranging from 3 to 30 minutes.

Test scores for delayed recall. The tests included under the Verbal delayed recall, Story delayed recall, and Visual delayed recall cognitive abilities were included under the Delayed recall ability. Additional tests included measures of Verbal short delayed free recall: total number of words recalled on trial A6 from the Rey Auditory 
Verbal Learning Test (max. score 15), total number of words recalled on the word list from the Consortium to Establish a Registry for Alzheimer's Disease after a 3 minute delay (max. score 10), and the short delayed free recall score from the California Verbal Learning Test (max. score 16), measures of Verbal short delay cued recall and long delayed cued recalled scores from the California Verbal Learning Test (max. score 16), and measures of Visual short delayed recall provided by the 3 minute delayed recall reproduction of the Rey-Osterrieth Complex Figure Test, measured as the total number of correct details recalled.

Semantic Memory refers to knowledge of previously learned verbal information that is now context-free and stored in long-term memory.

Test scores for semantic memory. Tests that measure knowledge for previously learned verbal information include vocabulary tests which are all multiple choice tests scored as total items correct (Extended Range Vocabulary Test, Mill Hill A and B, and a vocabulary task from McFall et al. 2010) and general information/fact recall questions with one correct answer scored as total number of items correct (Information from WAIS-R and a Fact Recall task from McFall et al. 2010). 


\section{APPENDIX B}

\section{Meta-analysis Code Book}

\section{Records}

There were three Excel sheets to record information from studies: two for tests scores by ability (one for diabetics versus non-diabetic controls and one for subgroups of diabetics (moderator variables) and one for tests by tests (diabetics versus non-diabetic controls). There is one Excel sheet that is the master list of test classification to a specified cognitive ability with reference for choice and page number documented.

There were individual sheets for every study coded: one for the diabetic and nondiabetic comparison and one for the moderator variables. If a study looked at diabetic versus non-diabetic controls and a moderator variable there are separate individual coding sheets for each condition; therefore some studies have more than one individual coding sheet based on what was done in the study. The labels below match what is found in the excel sheets and the explanations that follow them were used to fill every cell in the Excel sheets.

\section{Detailed Explanations of How Each of the Coding Sheets Was Completed}

\section{Individual Study Sheets}

Blank D vs. Non-D. Coding for studies included in the diabetic vs. non-diabetic metaanalysis

Citation and year: Last name of lead author and publication year Journal: The journal the study is published in Discipline: The Discipline/profession of the authors, if available Country: Country where participants were recruited

$\mathrm{N}$ diabetic group: number of the diabetic participants $\mathrm{N}$ control group: number of the control group participants \%male DG: \% of diabetic participants that were male 
\%male CG: \% of control participants that were male

Study design: Population (cross-sectional or longitudinal) or case-control (cross-sectional or longitudinal); only data from baseline (time 1) from longitudinal studies was included in the meta-analysis. Sampling/recruiting method used was recorded.

Study design code: 1 P, CS; 2 P, L; 3 CC, CS ; 4 CC, L

Subjects overlapping with another study: If overlap was declared in the article it was recorded and the study with the largest sample was included in the meta-analysis and all other studies with the same participants was excluded from the meta-analysis.

Age DG: Mean or median age of diabetic participants with SD

Age CG: Mean or median age of control participants with SD

Education DG: Mean or median number of years of education with SD for diabetic participants

Education CG: Mean or median number of years of education with SD for control participants

How diabetes operationalized: How the diabetes diagnosis was established in the study Inclusion criteria: listed all the inclusion criteria of the study Exclusion criteria: listed all the exclusion criteria of the study How excluded dementia: Put available information for how dementia was excluded, if dementia was not excluded, study cannot be included in the meta-analysis

Scores adjusted or matched: listed what scores were adjusted for or matched in each of the studies, only for the means, not for any of the statistical tests

HbA1c DG: Mean of median HbA1c level with SD of the diabetic participants HbA1c CG: Mean of median HbA1c level with SD of the control participants if reported

Fasting glucose DG: Mean or median fasting glucose level with SD of the diabetic participants

Fasting glucose CG: Mean or median fasting glucose level with SD of the control participants if reported

Diabetes duration DG: Mean or median with SD number of years since diagnosis for diabetic participants

Treatment modality: \% in DG (diet and exercise, medication, insulin injections, or combination)

BMI DG: BMI in diabetic participants if reported

BMI CG: BMI in control participants if reported 
Cholesterol DG: level of cholesterol in diabetic participants if reported

Cholesterol CG: level of cholesterol in control participants if reported

Triglycerides DG: level of triglycerides in diabetic participants if reported

Triglycerides CG: level of triglycerides in control participants if reported

Depression DG: \% of diabetic participants with depression if reported

Depression CG: \% of control participants with depression if reported

Hypertension DG: \% of diabetic participants with hypertension if reported

Hypertension CG: \% of control participants with hypertension if reported

Retinopathy DG: \% of diabetic participants with retinopathy if reported

Retinopathy CG: \% of control participants with retinopathy if reported

Atherosclerosis DG: \% of diabetic participants with atherosclerosis if reported

Atherosclerosis CG: \% of control participants with atherosclerosis if reported

Neuropathy DG: \% of diabetic participants with neuropathy if reported

Neuropathy CG: \% of control participants with neuropathy if reported

Scores Reported by test

Test: Name of the neuropsychological test administered

Score: Name of the score given from the test

Data: raw data from the studies, Means and SD for diabetic and control group (pooled group SD was used to calculate Cohen's $d$ as recommended by Hunter and Schmidt, 2004) or $t$ or $F$ values. How each column was completed when data were in MEANS and $\mathrm{SD}$ is below.

DG SD: diabetes group standard deviation

DG N: diabetes group $\mathrm{n}$, number of people in the diabetes group

CG SD: control group standard deviation

CG N: control group n, number of people in the control group

DG M: mean for the diabetes group

CG M: mean for the control group

Pooled SD: excel formula, didn't need to fill this in. Pooled SD of control group and diabetes group weighed for sample size

Cohen's $d$ : excel formula, didn't need to fill this in. Is the effect size for every score

filled into the summary spread sheet and was used to calculate the average Cohen's $d$ that were weighed for sample size based on the classification from the master list.

Add negative sign: if higher scores represented worse performance, put a yes in this column for that test score

Ability classification from original study: the ability or domain the score was classified under in the original study 
Ability classification from the master list: Ability the test score was assigned from the master list, not done for the test-by-test analyses

Cohen's d included in the ability average effect size (yes/no): if score effect size was included in the average effect size for that ability indicated yes, if not indicate no Cohen's d included in the test by test average effect size (yes/no): if score effect size was included in the average effect size for that test indicated yes, if not indicate no

For test by test analyses: Includes test used in each study based on total/summary scores for that test. Total/summary score was the score that represents the most general score given from a particular test. For test without summary scores the most salient score from each test was used.

\section{Individual Study Sheets}

Blank Mod. Var. Ana. Coding for studies included in one of the moderator variable meta-analyses

Diabetes group of interest either: older diabetics $>65$ years old, diagnosed clinically significant depression or diagnosed hypertension, longer diabetes duration $>5$ years since diagnosis, poorly controlled (above target HbA1c $(7.0$ is target)), insulin dependent (treated with insulin injections), diagnosed complications (atherosclerosis or retinopathy), studies published from 2006-2011 inclusive were Coded as 1 and were G1 for a given moderator variable.

Diabetes control group either: younger diabetics 40-65 years old, no diagnosed depression, no diagnosed hypertension, shorter diabetes duration, $<5$ years since diagnosis, well controlled (target HbA1c (7.0 is target)), non-insulin dependent (treated with diet and exercise or hypoglycemic medications), no diagnosed complications (atherosclerosis or retinopathy), Studies published from 2000-2005 inclusive were Coded as 0 and were G0 for a given moderator variable.

Moderator Variable: the moderator variable included in the study either age, depression, hypertension, diabetes duration, HbA1c level, insulin dependent vs non-insulin dependent, or complications (atherosclerosis or retinopathy)

Citation and year: Last name of lead author and publication year Journal: The journal the study is published in Discipline: The Discipline/profession of the authors, if available Country: Country where participants were recruited

N G1: number of coded 1 participants

N G0: number of coded 0 participants

$\%$ male G1: \% of coded 1 participants that are male

$\%$ male G0: \% of coded 0 participants that are male 
Study design: Population (cross-sectional or longitudinal) or case-control (cross-sectional or longitudinal); only data from baseline (time 1) from longitudinal studies was included in the meta-analysis. Sampling/recruiting method used was recorded.

Study design code: 1 P, CS; 2 P, L; 3 CC, CS ; 4 CC, L

Subjects overlapping with another study: If overlap was declared in the article it was recorded and the study with the largest sample was included in the meta-analysis and all other studies with the same participants was excluded from the meta-analysis.

Age G1: Mean or median age of coded 1 participants with SD

Age G0: Mean or median age of coded 0 participants with SD

Education G1: Mean or median number of years of education with SD for coded 1 participants

Education G0: Mean or median number of years of education with SD for coded 0 participants

How diabetes operationalized: How the diabetes diagnosis was established in the study Inclusion criteria: listed all the inclusion criteria of the study Exclusion criteria: listed all the exclusion criteria of the study How excluded dementia: Put available information for how dementia was excluded, if dementia was not excluded, study cannot be included in the meta-analysis

Scores adjusted or matched: listed what scores were adjusted for or matched in each of the studies, only for the means, not for any of the statistical tests

HbA1c G1: Mean of median HbA1c level with SD of the coded 1 participants HbA1c G0: Mean of median HbA1c level with SD of the coded 0 participants

Fasting glucose G1: Mean or median fasting glucose level with SD of the coded 1 participants

Fasting glucose G0: Mean or median fasting glucose level with SD of the coded 0 participants

Diabetes duration G1: Mean or median number of years since diabetes diagnosis with SD for coded 1 participants

Diabetes duration G0: Mean or median number of years since diabetes diagnosis with SD for coded 0 participants

Treatment modality: \% in each group (diet and exercise, medication, insulin injections, or combination)

BMI G1: BMI in diabetic participants if reported

BMI G0: BMI in control participants if reported 
Cholesterol G1: level of cholesterol in diabetic participants if reported

Cholesterol G0: level of cholesterol in control participants if reported

Triglycerides G1: level of triglycerides in diabetic participants if reported

Triglycerides G0: level of triglycerides in control participants if reported

Diagnosed complications: atherosclerosis or retinopathy

Diagnostic criteria used and whether depression was treated or not treated: this will be recorded

Diagnostic criteria used and whether hypertension was treated or not treated: this will be recorded

Scores Reported by test

Test: Name of the neuropsychological test administered

Score: Name of the score given from the test

Data: raw data from the studies, Means and SD for coded 1 and coded 0 groups ( pooled group SD was used to calculate Cohen's $d$ as recommended by Hunter and Schmidt, 2004) or $t$ or $F$ values. How each column was completed when data were in MEANS and SD is below.

G1 SD: diabetes group standard deviation

G1 N: diabetes group n, number of people in the diabetes group

G0 SD: control group standard deviation

G0 N: control group n, number of people in the control group

G1 M: mean for the diabetes group

G0 M: mean for the control group

Pooled SD: excel formula, didn't need to fill this in. Pooled SD of G1 and G0 weighed for sample size

Cohen's $d$ : excel formula, didn't need to fill this in. Is the effect size for every score filled into the summary spread sheet and was used to calculate the average Cohen's $d$ that were weighed for sample size based on the classification from the master list. Add negative sign: if higher scores represented worse performance, put a yes in this column for that test score

Ability classification from original study: the ability or domain the score was classified under in the original study

Ability classification from the master list: Ability the test score was assigned from the master list, not done for the test-by-test analyses

Cohen's d included in the ability average effect size (yes/no): if score effect size was included in the average effect size for that ability indicated yes, if not indicate no 


\section{Summary Sheets}

\section{D vs Non-D AbilityC}

-For each study: filled in across the row all of the corresponding information from the individual coding sheet

-Articles entered in alphabetical order by last name of the lead author

-Individual Cohen's $d$ for every ability present in each study filled in and Average $d$ values were calculated from these

-This sheet documented $\mathrm{n}$ for each ability and number of studies that measured any given ability

\section{D vs Non-D TbyT}

-For each study: filled in across the row all of the corresponding information from the individual coding sheet -Articles entered in alphabetical order by last name of the lead author -Cohen's $d$ for each test that was used at least 5 times across studies filled in and Average $d$ values were calculated from these

-This sheet documented $\mathrm{n}$ for each test and number of studies that used any given test

\section{Mod. Var. Ana. AbiliyC}

-For each study filled in across the row all of the corresponding information from the individual coding sheet

-Summary sheet was organized by moderator variable for clarity -Individual articles entered in alphabetical order under each moderator variable -Individual Cohen's $d$ for every ability present in each study filled in and Average $d$ values were calculated from these

-This sheet documented $\mathrm{n}$ for each ability and number of studies that measured any given ability for each moderator variable

\section{Master List}

\section{AbilityC Master List}

Test: Name of the neuropsychological test administered Score: Name of the score given from the test

Ability classification: Ability the test score was assigned from the list of possible cognitive abilities

Glossary ability classification: Consolidated classification used to calculate Effect Sizes Summary effect Sizes: Classification if score was used to calculate a summary effect size

Reference: Reference used to classify the score by ability either Strauss, Lezak, or the original article's classification

Page \#: page \# in Strauss or Lezak supporting test score ability classification Reason for not including a score: rationale for not including scores that were not included in the cognitive ability classification 


\section{REFERENCES}

References marked with an asterisk indicate studies included in the meta-analysis.

*Abbatecola, A.M., Lattanzio, F., Spazzafumo, L., Molinari, A.M., Cioffi, M., Canonico, R., ...Paolisso, G. (2010). Adiposity predicts cognitive decline in older persons with diabetes: a 2-year follow-up. PLOS ONE, 5, e10333.

doi:10.1371/journal.pone.0010333

Anderson, R.J., Freedland, K.E., Clouse, R.E. \&Lustman, P.J. (2001). The prevalence of comorbid depression in adults with diabetes. Diabetes Care, 24, 1069-1078.

Arvanitakis, Z., Wilson, R.S. \& Bennett, D.A. (2006). Diabetes mellitus, dementia, and cognitive function in older persons. Journal of Nutrition, Health and Aging, 10, $287-291$.

*Arvanitakis Z, Wilson, R.S., Bienias, J.L. Evans, D.A., \& Bennett, D.A. (2004). Diabetes mellitus and risk of Alzheimer disease and decline in cognitive function. Archives of Neurology, 61, 661-666.

*Asimakopoulou, K.G., Hampson, S.E., \&Morrish N.J. (2002). Neuropsychological functioning in older people with type 2 diabetes: The effect of controlling for confounding factors. Diabetic Medicine, 19, 311-316.

Awad, N., Gagnon, M. \& Messier, C. (2004). The relationship between impaired glucose tolerance, type 2 diabetes, and cognitive function. Journal of Clinical and Experimental Neuropsychology, 26, 1044-1080.

Baker, W. L., White, C. M., Cappelleri, J. C., Kluger, J., \& Coleman, C. I. (2009). 
Understanding heterogeneity in meta-analysis: The role of meta-regression. International Journal Of Clinical Practice, 63, 1426-1434. doi: 10.1111/j.17421241.2009.02168.x

*Bent, N., Rabbitt, P., \& Metcalfe, D. (2000). Diabetes mellitus and the rate of cognitive ageing. British Journal of Clinical Psychology, 39, 349-362.

Biessels, G. J., Deary, I. \& Ryan, C.M. (2008). Cognition and diabetes: A lifespan perspective. Lancet Neurology, 7, 184-190.

Biessels, G.J., van der Heide, L.P., Kamal, A., Bleys, R.L.A.W., \&Gispen, W.H. (2002).Ageing and diabetes: Implications for brain function. European Journal of Pharmacology, 441, 1-14.

Blazer, D.G., Moody-Ayers, S., Craft-Morgan, J., \& Bunchett, B. (2002). Depression in diabetes and obesity racial/ethnic/gender issues in older adults. Journal of Psychosomatic Research,53, 913-916.

Boyle, J.P., Honeycutt, A.A., Narayan, K.M.V., Hoerger, T.J., Geiss, L.S. , Chen, H. \& Thompson, T.J. (2001). Projection of diabetes burden through 2050 - Impact of changing demography and disease prevalence in the US. Diabetes Care, 24, 19361940.

Brands, A.M.A., van den Berg, E. Manshot, S.M., Biessels, G.J., Kappelle, L.J., De Haan, E.H.F. \& Kessels, R.P.C. (2007). A detailed profile of cognitive dysfunction and its relation to psychological distress in patients with type 2 diabetes mellitus. Journal of the International Neuropsychological Society, 13, 288-297. doi: 10.1017/S1355617707070312

Brands, A.M.A., Biessels, G.J., De Hann, E.H.F., Kappelle, L.I. \& Kessels., R.P.C. 
(2005). The effects of type1diabetes on cognitive performance - A meta-analysis. Diabetes Care, 28, 726-735.

Bruce, D.G., Davis, W.A. Casey, G.P., Starkstein, S.E., Clarnette, R.M., Almeida, O.P. \& Davis, T.M.E. (2008). Predictors of cognitive decline in older individuals with diabetes. Diabetes Care,31, 2103-2107. doi: 10.2337/dc08-0562

*Bruehl, H., Rueger, M., Dziobek, I., Sweat, V., Tirsi, A., Javier, E., ...Convit, A. (2007). Hypothalamic-pituitary-adrenal axis dysregulation and memory impairments in type 2 diabetes. Journal of Clinical Endocrinology \& Metabolism, 92, 2439-2445. doi: 10.1210/jc.2006-2540

Canadian Diabetes Association (CDA) (2011). The prevalence and costs of diabetes. Retrieved January $27^{\text {th }} 2011$ from: http://www.diabetes.ca/diabetes-andyou/what/prevalence/

*Christman, A.L., Vannorsdall, T.D., Pearlson, G.D., Hill-Briggs, F., \& Schretlen, D.J. (2010). Cranial volume, mild cognitive deficits, and functional limitations associated with diabetes in a community sample. Archives of Clinical Neuropsychology, 25, 49-59. doi:10.1093/arclin/acp091

*Cosway, R., Strachan, W.J., Dougall, A., Frier, B.M. \& Dreary I.J. (2001). Cognitive function and information processing in type 2 diabetes. Diabetic Medicine, 18, 803-810.

Cukierman, T., Gerstein, H.C. \& Williamson, J.D. (2005). Cognitive decline and dementia in diabetes - systematic overview of prospective observational studies. Diabetologia, 48, 2460-2469. doi: 10.1007/s00125-005-0023-4

Defronzo, R.A, Bonadonna, R.C. \&Ferrannini, E. (1997).Pathogenesis of NIDDM. In: 
Alberti, K.G.M.M., Zimmet, P., DeFronxo, R.A. \& Keen, H. eds. International textbook of diabetes mellitus. $2^{\text {nd }}$ ed. New York: Wiley, 635-711.

Ebady, S.A., Arami, M.A. \&Shafigh, M.H. (2008). Investigation on the relationship between diabetes mellitus type 2 diabetes and cognitive impairment. Diabetes Research and Clinical Practice, 83, 305-309. dio: 10.1016/j.diabres.2008.08.020

*Espeland, M.A., Miller, M.E., Goveas, J.S., Hogan, P.E., Coker, L.H., Williamson, J., ...Resnick, S.M. (2011). Cognitive function and fine motor speed in older women with diabetes mellitus: results from the women's health initiative study of cognitive aging. Journal of Women's Health, 20, 1435-1443. DOI: 10.1089/jwh.2011.2812

*Fuh, J.L., Wang, S.J., Hwu, C.M., \& Lu, S.R. (2007). Glucose tolerance status and cognitive impairment in early middle-aged women. Diabetic Medicine, 24, 788791. DOI: 10.1111/j.1464-5491.2007.02170.x

*Gallacher, J.E., Pickering, J., Elwood, P.C., Bayer, A.J., Yarnell, J.W., \& Ben-Shlomo, Y. (2005). Glucoregulation has greater impact on cognitive performance than macro-vascular disease in men with type 2 diabetes: data from the Caerphilly study. European Journal of Epidemiology, 20, 761-768. DOI 10.1007/s10654005-2146-9

Gaudieri, P. A., Chen, R., Greer, T.F. \&Holmes, C.S. (2008). Cognitive function in children with type 1 diabetes. Diabetes Care, 31, 1892-1897. doi: 10.2337/dc072132

*Haroon E, Watari K, Thomas A, Ajilore O, Mintz J, Elderkin-Thompson V, ... Kumar, 
(2009). Prefrontal myo-inositol concentration and visuospatial functioning among diabetic depressed patients. Psychiatry Research, 171, 10-19.

doi:10.1016/j.pscychresns.2008.03.006

Hassing, L.B., Hofer, S.M., Nilsson, S.E., Berg, S,. Pedersen, N.L., McClearn, G. \& Johansson, B. (2004). Comorbid type 2 diabetes mellitus and hypertension exacerbates cognitive decline: evidence from a longitudinal study. Age and Ageing, 33, 355-361. doi: 10.1093/ageing/afh100

Hedges, L.V. \&Olkin, I. (1985).Statistical methods for meta-analysis. Orlando, FL: Academic Press.

*Hewer, W., Mussell, M., Rist, F., Kulzer, B., \& Bergis, K. (2003). Short-term effects of improved glycemic control on cognitive function in patients with type 2 diabetes. Gerontology, 49, 86-92. DOI: 10.1159/000067947

Holmes, C.T. (1984). Effect size estimation in meta-analysis. Journal of Experimental Education, 52,106-109.

Hunter, J.E. \& Schmidt, F.L. (2004).Methods of meta-analysis: correcting error and bias in research findings. Thousands Oaks, CA: Sage Publications Ltd.

Hussain, A., Claussen, B., Ramachandran, A. \& Williams, R. (2007).Prevention of type 2 diabetes: A review. Diabetes Research and Clinical Practice, 76, 317-326. doi: 10.1016/j.diabres.2006.09.020

Keefover, R.W. (1998). Aging and cognition. Neurologic Clinics, 16, 635-648.

Kolt, C.T. \&Seaquist, E.R. (2008). Cognitive dysfunction and diabetes mellitus. Endocrine Reviews, 29, 494-511. doi: 10.1210/er.2007-0034

*Kumar, R., Anstey, K.J., Cherbuin, N., Wen, W., \& Sachdev, P.S. (2008). Association 
of type 2 diabetes with depression, brain atrophy, and reduced fine motor speed in a 60- to 64-year-old community sample. American Journal of Geriatric Psychiatry, 16, 989-998.

Kumari, M., Brunner, E. \& Fuhrer, R. (2000). Minireview: Mechanisms by which the metabolic syndrome and diabetes impair memory. The Journals of Gerontology, 55, 228-232.

Lezak, M.D., Howieson, D.B. \&Loring, D.W. (2004). Neuropsychological Assessment.( $4^{\text {th }}$ Ed.). Oxford, Oxford University Press

Manshot, S.M., Biessels, G.J., de Valk, H., Algra, A., Rutten, G.E.H.M., van der Grond, J. \& Kappelle, L.J. (2007). Metabolic and vascular determinants of impaired cognitive performance and abnormalities on brain magnetic resonance imaging in patients with type 2 diabetes. Diabetologia, 50, 2388-2397. doi: 10.1007/s00125007-0792-Z

Manschot, S.M., Brands, A.M., van der Grond, G.J., Kessels, R.P.C., Algra, A., Kappelle, L.J. \& Biessels, G.J. (2006). Brain magnetic resonance imaging correlates of impaired cognition in patients with type 2 diabetes. Diabetes, 55, 1106-1113.

*McFall, G.P., Geall, P.B., Fischer, A.L., Dolcos, S. \&Dixon, R.A. (2010). Testing Covariates of Type 2 Diabetes-Cognition Associations in Older Adults: Moderating or Mediating Effects? Neuropsychology, 24, 547-562. doi: 10.1037/a0019246

*Mogi, N., Umegaki, H., Hattori, A., Maeda, N., Miura, H., Kuzuya, M., ... Iguchi, A. 
(2004). Cognitive function in Japanese elderly with type 2 diabetes mellitus. Journal of Diabetes \& its Complications, 18, 42-46. doi:10.1016/S10568727(03)00078-3

Monette, M.C.E. (2012). What can clinical neuropsychology offer type II diabetes mellitus treatment management? Health Science Inquiry, 3, 53-54.

Munch, G, Schinzel, R., Loske, C., Wong, A., Durany, N., Li, J.J., ... Riederer, P. (1998). Alzheimer's disease: Synergistic effects of glucose deficit, oxidative stress and advanced glycation endproducts. Journal of Neural Transmission, 105,439-461.

Munshi, M., Grande, L., Hayes, M., Ayres, D., Suhl, E., Capelson, R., ...Weinger, K. (2006). Cognitive dysfunction is associated with poor diabetes control in older adults. Diabetes Care, 29, 1794-1799.doi: 10.2337/dc06-0506

Murthy, S.B., Jawaid, A. \& Schulz, P.E. (2008). Diabetes mellitus and dementia: Advocating an annual cognitive screening in patients with diabetes mellitus. The Journal of the American Geriatrics Society, 56, 1976-1977.

National Research Council. (1992). Combining information: Statistical issues and opportunities for research. Washington DC: National Academy of Sciences Press. In: Hunter, J.E. \& Schmidt, F.L. (2004). Methods of meta-analysis: correcting error and bias in research findings. Thousands Oaks, CA: Sage Publications Ltd.

Okereke, O.I., Kang, J.H., Cook, N.R., Gaziano, J.M., Manson, J.E., Buring, J,E. \& Grodstein, F. (2008). Type 2 diabetes mellitus and cognitive decline in two large cohorts of community-dwelling older adults. Journal of the American Geriatrics Society, 56, 1028-1036. doi: 10.1111/j.1532-5415.2008.01686.x

Ott, A., Stolk, R.P., van Harskamp, F., Pols, H.A.P., Hofman, A., \&Breteler, M.M.B. 
(1999). Diabetes mellitus and the risk of dementia. Neurology, 53, 1937-1942.

Pradhan, A. (2007). Obesity, metabolic syndrome, and type 2 diabetes: Inflammatory basis of glucose metabolic disorders. Nutrition Reviews, 65, 152-156. doi:

10.1301/nr.2007.dec.S152-S156

Pirttila, T., Jarvenpaa, R., Laippala, P., Frey H. (1992). Brain atrophy on computerized axial tomography scans: Interaction of age, diabetes, and general morbidity. Gerontology, 38, 285-291.

Ryan, C.M. (2006). Diabetes and brain damage: more (or less) than meets the eye? Diabetologia,49, 2229-2233. doi: 10.1007/s00125-006-0392-3

Ryan, C.M., Freed, M.I., Rood, J.A, Cobitz, A.R., Waterhouse, B.R. \& Strachan, M.W.J. (2006). Improving metabolic control leads to better working memory in adults with type 2 diabetes. Diabetes Care, 29, 345-351

*Ryan, C.M. \& Geckle, M. (2000a). Circumscribed cognitive dysfunction in middleaged adults with type 2 diabetes. Diabetes Care, 23, 1486-1493.

Ryan, C.M. \& Geckle, M. (2000b). Why is learning and memory dysfunction in type 2 diabetes limited to older adults? Diabetes-Metabolism Research and Reviews, 16, 308-315.

Ryan, C.M., Williams, T.M., Orchard, T.J. \& Finegold, D.N. (1992). Psychomotor slowing is associated with distal symmetrical polyneuropathy in adults with diabetes mellitus. Diabetes, 41, 107-113.

Saczynski, J.S., Jonsdottir, M.K., Garcia, M.E., Jonsson, P., Peila, R. Eiriksdottir, G., 
...Launer, LJ. (2008). Cognitive impairment: An increasingly important complication of type 2 diabetes. American Journal of Epidemiology, 168, 11321139. doi: 10.1093/aje/kwn228

Sastre, A.A. \&Grimley, E.J. (2003). Effect of the treatment of type II diabetes mellitus on the development of cognitive impairment and dementia (Review). Cochrane Database of Systematic Reviews, 1, 1-20.

Singh, R., Barden, A., Mori, T.\& Beilin, L.(2001). Advanced glycation end-products: A review. Diabetologia, 44, 129-146.

Rabin, L.A, Wishart, H. A., Fields R.B, \&Saykin, A.J. (2006). In Snyder, P. J., Nussbaum, P. D., \& Robins, D. L. (Eds.) Clinical Neuropsychology - A Pocket Handbook for Assessment. Washington, DC: American Psychological Association.

Sommerfield, A. J., Dreary, I.J. \&Frier, B.M. (2004). Acute hyperglycemia alters mood states and impairs cognitive performance in people with type 2 diabetes. Diabetes Care, 27, 2335-2340.

Strauss, E., Sherman, E.M.S., \&Spreen, O. (2006). A compendium of neuropsychological tests: Administration, norms, and commentary. (3rd. ed.). New York: Oxford University Press.

Testa, M.A. \& Simonson, D.C. (1998). Health economic benefits and quality of life during improved glycemic control in patients with type 2 diabetes mellitus: A randomized, controlled, double-blind trial. Journal of the American Medical Association, 280, 1490-1496.

Tisserand, D.J. \&Jolles, J. (2003). On the involvement of prefrontal networks in cognitive 
ageing. Cortex, 39, 1107-1128.

*Toro, P., Schonknecht, P., \& Schroder, J. (2009). Type II diabetes in mild cognitive impairment and Alzheimer's disease: Results from a prospective population-based study in Germany. Journal of Alzheimer's Disease, 16, 687-691. DOI 10.3233/JAD-2009-0981

van den Berg, E, Kloppenborg, R.P., Kessels R.P.C., Kappelle, L.J. \&Biessels, G.J. (2009). Type 2 diabetes mellitus, hypertension, dyslipidemia and obesity: A systematic comparison of their impact on cognition. Biochimica et Biophysica Acta, 1792, 470-481.

*van den Berg, E., Reijmer, Y.D., de Bresser, J., Kessels, R. P. C., Kappelle, L. J. \& Biessels, G. J. (2010). A 4 year follow-up study of cognitive functioning in patients with type 2 diabetes mellitus. Diabetologia, 53, 58-65. doi: $10.1007 / \mathrm{s} 00125-009-1571-9$

*van Elderen, S.G., de Roos, A., de Craen, A.J., Westendorp, R.G., Blauw, G.J., Jukema, J.W., ... van der Grond, J. (2010). Progression of brain atrophy and cognitive decline in diabetes mellitus: a 3-year follow-up. Neurology, 75, 997-1002.

*van Harten, B., Oosterman, J., Muslimovic, D., van Loon, B-J.P., Scheltens, P. \& Weinstein, H.C. (2007). Cognitive impairment and MRI correlates in the elderly patients with type 2 diabetes mellitus. Age and Aging, 36, 164-170. doi: 10.1093/ageing/afl180

Vitek, M.P., Bjattacharya, K., Glendening, M.J., Tonge, P., Yi, Y.L., Stopa, E.,...Cerami, 
A. (1994). Advanced glycation end products contribute to amyloidosis in Alzheimer's disease. Proceedings of the National Academy of Sciences, 91, 47664770.

*Wahlin, A., Nilsson, E., \& Fastbom, J. (2002). Cognitive performance in very old diabetic persons: The impact of semantic structure, preclinical dementia, and impending death. Neuropsychology, 16, 208-216. DOI: 10.1037//08944105.16.2.208

Waldstein, S.R. (2003). The relation of hypertension to cognitive function. Current Directions in Psychological Science, 12, 9-12.

*Watari, K., Elderkin-Thompson, V., Ajilore, O., Haroon, E., Darwin, C., Pham, D., ... Kumar, A. (2008). Neuroanatomical correlates of executive functioning in depressed adults with type 2 diabetes. Journal of Clinical \& Experimental Neuropsychology: Official Journal of the International Neuropsychological Society, 30, 389-397. DOI: 10.1080/13803390701440486

Watari, K., Letamendi, A., Elderkin-Thompson, V., Haroon, E., Miller, J., Darwin, C. \& Kumar, A. (2006).Cognitive function in adults with type 2 diabetes and major depression. Archives of Clinical Neuropsychology, 21, 787-796. doi: 10.1016/j.acn.2006.06.014

Whitmer, R.A. (2007). Type 2 diabetes and risk of cognitive impairment and dementia. Current Neurology and Neuroscience Reports, 7, 373-380.

*Yau, .PL., Javier, D., Tsui, W., Sweat, V., Bruehl, H., Borod, J.C., \& Convit, A. (2009). 
Emotional and neutral declarative memory impairments and associated white matter microstructural abnormalities in adults with type 2 diabetes. Psychiatry Research, 174, 223-230. doi:10.1016/j.pscychresns.2009.04.016

*Zhou, H., Lu, W., Shi, Y., Bai, F., Chang, J., Yuan, Y., ... Zhang, Z. (2010). Impairments in cognition and resting-state connectivity of the hippocampus in elderly subjects with type 2 diabetes. Neuroscience Letters, 473, 5-10. doi:10.1016/j.neulet.2009.12.057 


\section{VITA AUCTORIS}

NAME: $\quad$ Michelle C. E. Monette

PLACE OF BIRTH: Monetteville, Ontario

YEAR OF BIRTH: 1986

EDUCATION École Secondaire Sainte-Famille, Mississauga, Ontario 2000 - 2004

York University - Glendon Campus, Toronto, Ontario 2004 - 2009 B.A. Psychology

York University - Glendon Campus, Toronto, Ontario 2009 - 2010 B.A. Environmental and Health Studies

University of Windsor, Windsor, Ontario 2010 - 2012 M.A. Clinical Neuropsychology 